\title{
Full-Field and Single-Shot Full-Field Optical Coherence Tomography: A Novel Technique for Biomedical Imaging Applications
}

\author{
Hrebesh Molly Subhash \\ Division of Biomedical Engineering, Biophotonics \& Functional Imaging Laboratory, Oregon Health \& Science University, \\ Portland, OR 97239, USA \\ Correspondence should be addressed to Hrebesh Molly Subhash, hrebeshms@gmail.com
}

Received 17 October 2011; Revised 23 December 2011; Accepted 23 December 2011

Academic Editor: Joseph Rosen

Copyright () 2012 Hrebesh Molly Subhash. This is an open access article distributed under the Creative Commons Attribution License, which permits unrestricted use, distribution, and reproduction in any medium, provided the original work is properly cited.

Since its introduction, optical coherence tomography (OCT) technology has advanced dramatically in various field of both clinical and fundamental research. Full-field and Single-shot full-field OCT (FF-OCT and SS-FF-OCT) are alternative OCT concepts, which aims to improve the image acquisition speed and to simplify the optical setup of conventional point-scan OCT by realizing direct line field or full-field sample imaging onto an array or line detector such as CCD or CMOS camera. FF-OCT and SS-FFOCT are based on bulk optics Linnik-type Michelson interferometer with relatively high numerical aperture (NA) microscopic objectives. This paper will give you an overview of the principle of various types of FF-OCT and SS-FF-OCT techniques and its associated system design concept and image reconstruction algorithms.

\section{Introduction}

Optical coherence tomography (OCT) is a novel noninvasive optical imaging modality based on low-coherence interferometry. The first version of this technique was devised in 1990 by Dr. Naohiro Tanno, a professor at Yamagata University $[1,2]$, and then perfected in 1991 by Massachusetts Institute of Technology team headed by Professor James Fujimoto [3]. OCT can enable the noninvasive, noncontact imaging of cross-sectional structures in biological tissues and materials with high resolution. In principle, OCT is an optical analogue to clinical ultrasound. In OCT, the temporally gated back-reflected optical pulse remitted from scattering sites within the sample is localized by lowcoherence interferometry (LCI) [4-8]. This is typically achieved with a Michelson interferometer. The sample rests in one arm of the interferometer, and a scanning reference optical delay line comprises the other arm. In LCI, light interferes at the detector only when light reflected from the sample is matched in pathlength to light reflected from the scanning reference mirror. A single scan of the reference mirror thus provides a one-dimensional reflectivity profile of the sample. Two-dimensional cross-sectional images are formed by laterally scanning the incident probe beam across the sample. The reconstructed OCT images are essentially a map of the changes in the refractive index of refraction that occurs at internal interfaces. Just like ultrasound images, the discontinuities in acoustic impedance occur.

The principle difference between ultrasound and optical imaging is that the velocity of light is approximately a million times faster than the velocity of sound. For this reason, the distance within the materials or tissues with a resolution of $10 \mu \mathrm{m}$ by measuring the echo time delay of back-reflected or back-scattered light wave corresponds to a time resolution of 30 femto $\left(10^{-15}\right)$ seconds, which is well beyond the limits of currently possible direct electronic detection system. But for the ultrasound, the echo time delay is approximately $100 \mathrm{~ns}$, which can be easily realized with modern electronic detection systems. Thus, in OCT, the measurements of each time delay are based on correlation techniques, in which the back-scattered or back-reflected light is compared to the 
reference light with known path length. OCT uses the lowcoherence interferometric method to measure this echo time delay with dynamic range and sensitivity.

\section{Variant OCT Imaging Schemes}

In OCT, the coherence-gated information about the elementary volume of the scatters within the obscuring scattering specimen can be obtained from either the time-domain measurement principle (TD-OCT) or the frequency domain (FD-OCT) measurement principle $[9,10]$. In time-domain OCT, the wavenumber-dependent photo detector current is captured using a single or multiple detector where the reference mirror is scanned to match the optical path from reflections within the sample. Depending on the scanning modality and the signal detection scheme employed, the time-domain OCT can be further be classified into three: (1) point-scan (flying scan OCT), (2) line field (linear-OCT), and (3) full-field (wide-field) OCT. On the other hand, the signal detection in FD-OCT relies on the transformation of the OCT time-varying signal along the optical axis, termed the A-scan, into the frequency domain. Thus, FD-OCT has the advantage that the full-sample depth information is obtained in a parallel manner such that no moving parts are necessary. The basic physics behind FD-OCT is based on the inverse scattering theorem. According to Wiener-Khintchine theorem, the spectral power amplitude of the back-scattered wave equals the Fourier transform of the axial distribution of the object scattering potential. Recently, FD-OCT has been widely attracted in the biomedical imaging field due to its higher sensitivity and imaging speed compared to conventional TD-OCT. Based on the implementation, the FDOCT can be divided into two classes: spectral domain OCT (SD-OCT) and swept-source OCT (SS-OCT). In SDOCT, the optical frequency components are captured simultaneously with a dispersive element and linear detector, on the other hand in SS-OCT the optical frequency components are captured by a single detector in a time-encoded sequence by sweeping the frequency of the optical source. Recent studies have shown that Fourier domain OCT can provide signal to noise ratio that is more than $20 \mathrm{~dB}$ better than the conventional TD-OCT [11-13].

However, FD-OCT also has remarkable drawbacks. One limitation in frequency domain is the optical spectrum at the output of the interferometer consists of symmetric spectral terms, which cause same image result for positive and negative OPDs called mirroring effect. Different methods have been developed to eliminate this problem, such as phase shifting interferometry or complex signal processing. The simple detection in the frequency domain usually introduces spatial frequency ambiguity, autocorrelation artifacts, originating from internal interferences of the sample and the system as well as depth-dependent SNR loss, caused by finite spectral line width [14]. Furthermore, nonuniform FD sampling deteriorates TD signal quality and time-consuming postprocessing is a necessity for all FDOCT techniques. It was found that some of these effects can be largely compensated by multiple, phase-shifted imaging of the same region (i.e., A-scan), followed by reconstruction of the complex phase of the FD signal [15], resampling and transformation into the time-domain. Moreover, for getting better lateral resolution, the technique such as dynamic focusing, which is generally used in time domain OCT, is not easily implemented in FD-OCT. In traditional FD-OCT the imaging optics are devised with large depth of focus, to accommodate entire range of the A-scan, usually 1-2 mm and which limits the possibility of using high numerical aperture objective to enhance the transverse resolution. However, recently by employing Bessel beam with axicon lenses, the extended focal length FD-OCTs were demonstrated with cellular level resolution [16].

\section{Full-Field Optical Coherence Tomography}

Conventional point-scan OCT [17-19] can enable noninvasive, noncontact $3 \mathrm{D}$ cross-sectional imaging of structures in highly scattering specimens with very high resolution. However, for reconstructing the 3D image the conventional OCT system needs single-pointer raster-scanning scheme, in which the focus of the probing beam scans across the sample surface and detects the back-scattered light with a single element photo-detector. Thus, a fiber-optics-based point-scan time-domain OCT scheme requires at least three scans (one depth and two lateral scans) for acquiring threedimensional images. The lateral scans addresses laterally adjacent sample positions whereas the depth scan detects longitudinal depth positions of light-reflected sites in the sample. Major limitation in point scans OCT approach is the mechanical scanning system giving rise to motion artifacts due to mechanical jitter and limited repeatability. Full-field OCT (FF-OCT) [20, 21] and wide-field (WF-OCT) [22] OCT are alternative OCT concepts, which aim to improve the image acquisition speed and to simplify the optical setup of conventional point-scan OCT by realizing direct widefield or full-field sample imaging onto an array detector such as CCD or CMOS camera. FF-OCT is based on Linnikbased Michelson interferometer with relatively high identical numerical aperture (NA) microscopic objectives on both arms of the interferometer. Whereas, WF-OCT uses imaging optics with a single lens to image the large sample area, compromising the lateral resolution. Recently, FF-OCT has been of increased interest as a nonscanning, high-resolution, en face imaging method in several medical and non-medical applications. Moreover, en face image can provide new information, which may complement that provided by the longitudinal cross-sectional image [23].

3.1. Basic Principle of FF-OCT. The basic configuration of full-field OCT systems is based on a low-coherence interferometer with a CCD camera is placed at the output as detector. The CCD can enable the acquisition of $2 \mathrm{D}$ en face image in a single exposure without any lateral scanning. However, the direct use of CCD cameras for heterodyne detection in time domain is limited by the relatively slow frame rate of the commercially available CCD camera (typically $\approx 100 \mathrm{~Hz}$ for $512 \times 512$ pixels). Therefore, 


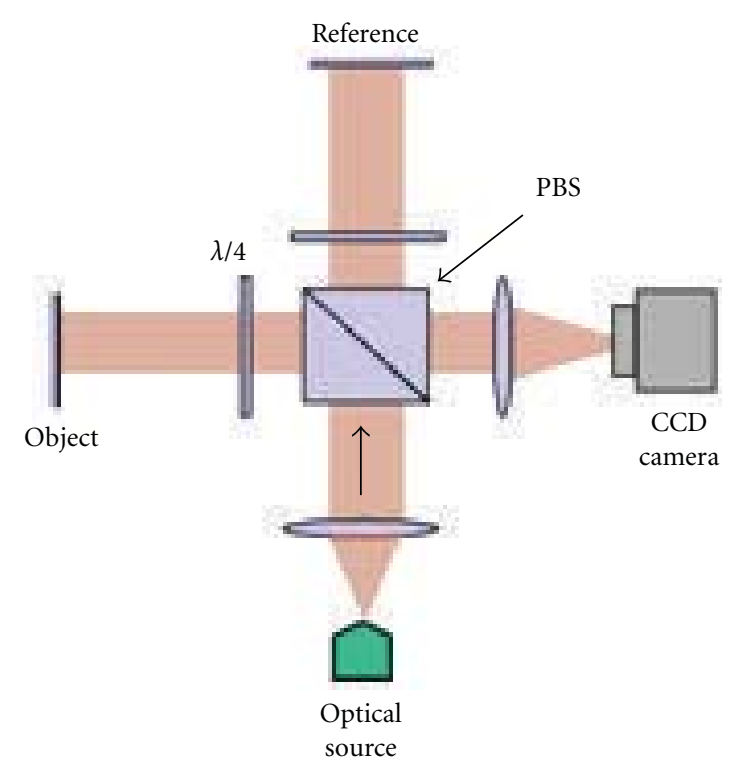

(a)

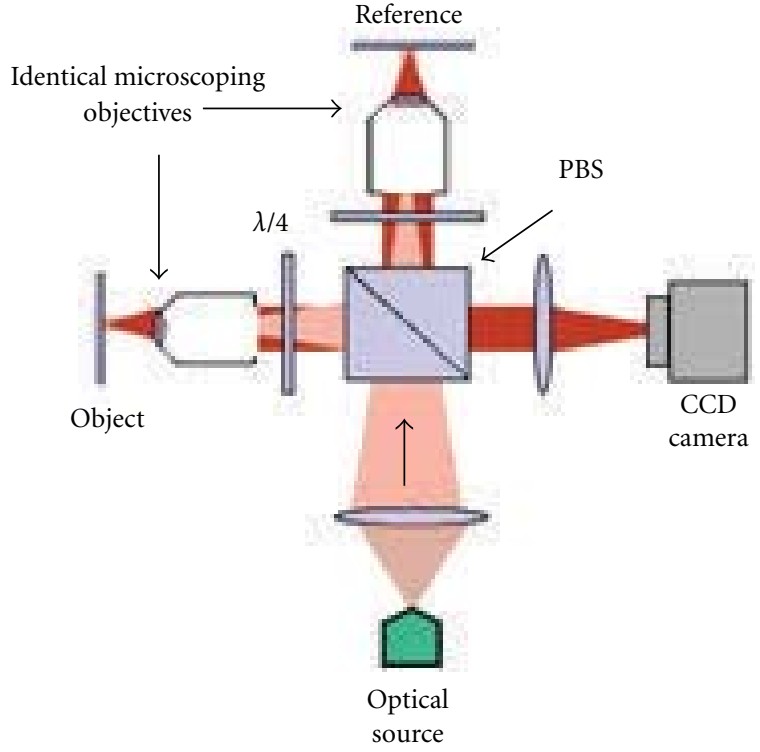

(b)

FIGURE 1: Typical full-field OCT configurations. (a) Michelson geometry for wide-field OCT (WF-OCT); (b) Linnik geometry for full-field OCT (FF-OCT).

the depth scan acquisition speed has to be drastically reduced in order to permit sufficient sampling of the interferometric signal. The temporal phase-stepping interferometric scheme is commonly used for the reconstruction of en face OCT image.

In FF-OCT, different interferometric configurations like Michelson [20], Mirau [24], or Linnik [25, 26] configuration can be used. For wide aperture system, the spherical aberration in the axial direction is very dominant for Michelson and Mirau configuration compared to Linnik. In case of Linnik configuration, optical pathlength and focusing in both arms of the interferometer can be adjusted independently [27]. Moreover, in Linnik configuration high numerical aperture (NA) objective can be used to obtain high spatial resolution. In general, the axial resolution of the tomographic image in FF-OCT depends on both coherence length of the source (coherence effect) and the N.A of the objective (longitudinal spatial coherence effect) [28]. A variant scheme of FF-OCT that utilizes objective with high N.A $(<0.4)$ is called full-field optical coherence microscopy (FF-OCM) and which is used for obtaining very high resolution $[29,30]$. In this scheme, the longitudinal coherence effect is more dominant than coherence effect. Figure 1 shows the basic setup of FF-OCT based on Michelson (Figure 1(a)) and Linnik configuration (Figure 1(b)).

FF-OCT scheme utilizes wide variety of optical sources, which can be based on either a source with spatially coherent illumination or spatially incoherent illumination. In FF-OCT, depending upon the nature of illumination, the multiply scattered light from the specimen can result in strong crosstalk-generated noise between parallel detection channels, thus each detector should ideally not detect light originating from outside its conjugate volume. To avoid crosstalk, one must prevent interference occurring between adjacent pixels at the detection plane [33]. This can be achieved by controlling the degree of spatial coherence of full-field illumination. FF-OCT utilizing spatially incoherent sources such as thermal sources can completely suppress the effect of coherent crosstalk [34]. Moreover, thermal can also provide very high axial resolution because of its broad spectral bandwidth. However, the brightness per spatial mode of thermal sources remains insufficient requiring a trade-off between sensitivity and acquisition speed. The major interest of spatially coherent illumination in FF-OCT resides in the possibility to use powerful pulsed lasers such as femtosecond lasers, which can allow higher sensitivity and acquisition speed than with thermal light sources. On the contrary, spatially coherent source can generate considerable amount of coherent crosstalk which degrade the image quality.

\subsection{Performances of FF-OCT. In FF-OCT, the characteristics} of the optical source determine the general performance of the system. For example, the short coherence length of the optical source determines the axial resolution and the nominal wavelength determines the achievable penetration depth, of the system. The larger the line width, $\Delta \lambda$ (1) of the source, the smaller the coherence length $l_{c}$ of the source. There are four main criteria that need to be considered when choosing a light source for OCT. These parameters are wavelength, bandwidth, single-transverse mode power, and stability. The depth of penetration in OCT is limited by both scattering and absorption of the sample. Absorption window for most biological tissues between $0.65 \sim 1.4 \mu \mathrm{m}$ has a local minimum in the absorption of water and blood in the tissues. Absorption attenuates the light in the imaging subject and is wavelength-dependent. Because more than 


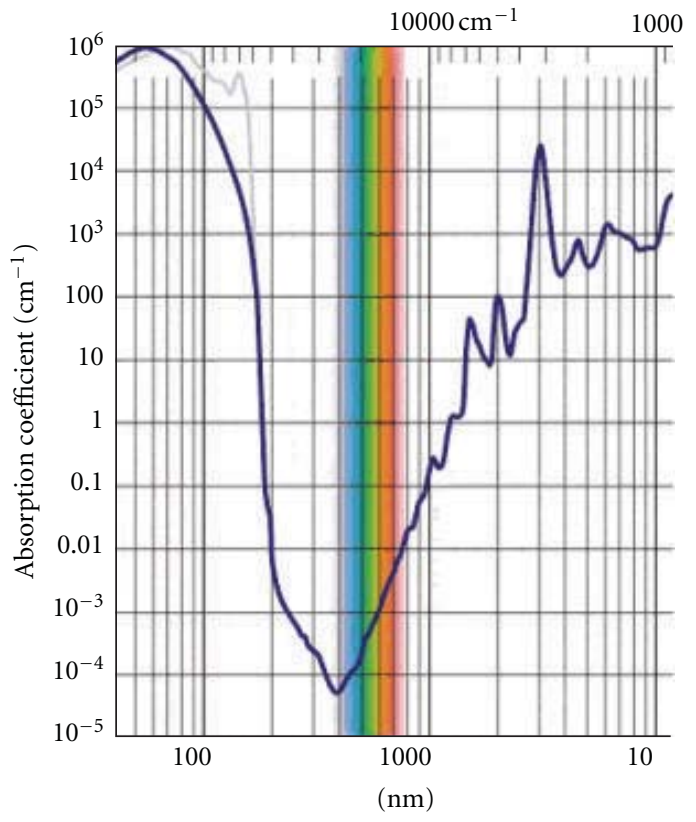

FIGURE 2: Water absorption spectrum. The original data consult from http://omlc.ogi.edu/spectra/water/index.html.

$80 \%$ of biological tissue consists of water, the absorption of tissue is mostly attributed to the water. If we analyze the water absorption spectrum shown in Figure 2, we can find a window with lower absorption, termed "therapeutic windows" for biomedical optics, existing around $600 \mathrm{~nm}$ to $800 \mathrm{~nm}$. Absorption by melanin, as well as the scattering from structure protein such as collagen and elastin, exhibits a nearly monotonic decrease with increasing wavelength over this region. These optical properties of tissues have been investigated to some degree both experimentally and by a number of analytical and numerical models. Therefore, the selection of wavelength is a very critical parameter in OCT.

3.2.1. Axial Resolution. Unlike in conventional optical microscopy, the apparent advantage of OCT is that the axial resolution is completely decoupled from the lateral resolution. The axial resolution of an OCT is an important specification for many biomedical applications. In many applications high axial resolution is required to distinguish different cellular ultrastructures and type. The axial resolution of an OCT system is generally defined as half the coherence length of the source spectrum $l_{c}$. The source coherence length $l_{c}$ is simply the coherence time multiplied by the speed of light. For a source with a Gaussian spectral distribution, the axial resolution is given by

$$
\Delta z=\frac{l_{c}}{2}=\frac{2 \ln (2)}{\pi} \frac{c}{\Delta \nu}=\frac{2 \ln (2)}{\pi} \frac{\lambda^{2}}{\Delta \lambda} \approx 0.44 \frac{\lambda_{0}^{2}}{\Delta \lambda},
$$

where $\Delta z$ and $\Delta \lambda$ are the full width at half maximum of the autocorrelation function and spectral width and $\lambda_{0}$ is the source center wavelength. It can be seen that broadband light sources are required to achieve high axial resolution, as the axial resolution is inversely proportional to the spectral width of the light source. The most popular light source currently used in OCT appears to be superluminescent diode (SLD). A typical SLD has a bandwidth $\Delta \lambda=20 \mathrm{~nm}$, which corresponds to a coherence length of $44 \mu \mathrm{m}$ and since $l_{c}$ is defied for a round trip, so this leads to a depth pixel size of $22 \mu \mathrm{m}$ in air, and in tissue, by considering the refractive index of 1.4 gives $16 \mu \mathrm{m}$. Modern sources for OCT use Kerrlens mode-locked lasers and photonic crystal fibers to achieve submicron meter coherence length. Apart from the wideline width, the sources for OCT have to exhibit a smooth Gaussian spectrum profile $[35,36]$.

3.2.2. Lateral Resolution. As conventional microscopy, the lateral resolution that may be achieved with OCT imaging is determined by the focus spot of the light beam and is completely decoupled from the axial resolution. There for the optical design of the lateral scanning does not correlate with the axial resolution. The lateral resolution $\Delta x$ can be written as

$$
\Delta x=\frac{4 \lambda}{\pi}\left(\frac{f}{d}\right)=\left(\frac{2 \lambda}{\pi}\right) \frac{1}{\mathrm{NA}},
$$

where $f$ is the focal length of the objective lens, and $d$ is the $1 / e^{2}$ Gaussian beam waist at the objective lens. It can be seen from the above equation that high lateral resolution can be achieved by using a large numerical aperture (NA) to decrease the spot size. However, for the selection of optics there is a trade-off between the lateral resolution and the imaging depth. A higher lateral resolution leads to a decrease in the depth of focus, or confocal parameter $b$, which is twice the Rayleigh range

$$
2 \Delta z_{R}=b=\frac{\pi \Delta x^{2}}{2 \lambda}
$$

The Rayleigh range gives the distance from the focal point to the point where the light beam diameter has increased by a factor of $\sqrt{2}$. This effectively limits the scanning range of the OCT, quite apart from the working range of the scanning reference delay line, as it is the range over which lateral resolution is maintained. Figure 3 shows the relation between the depth of focus (confocal parameter) and the focused spot size for the objective lens with high and low numerical aperture.

3.3. Image Reconstruction in FF-OCT. Over the past decade, several FF-OCT image reconstruction schemes were reported based on wide verity of optical configurations. Phase stepping and the type of PSI (phase-shifting interferometry) algorithm [37] were mainly used for the extraction of the interferometric signal. Beaurepaire et al. reported the first FF-OCT system implemented in a commercial microscope using multiplexed lock-in detection technique [20]. They use a fast photoelastic birefringence modulator to introduce a sinusoidal phase shift between sample and reference lights. Four images with the different phase shift are thus recorded successively to reconstruct the en face OCT image. FF-OCT based on conventional sequential phase shifting method was 
realized by several authors $[38,39]$. In this scheme the interferometer's reference arm or the sample is temporally shifted using a piezoelectric translation stage and then the sequentially acquired three or four-phase-stepped images were used for the reconstruction of en face OCT image. Akiba et al. used a pair of CCD cameras with fast liquid crystal shutters to realize the $2 \mathrm{D}$ heterodyne detection $[40,41]$. For in vivo subcellular level imaging, a dual-channel FF-OCT incorporated with a pair of CCD camera has been reported [40]. In this scheme, first, pair of quadraturely phase-shifted images are simultaneously captured using the CCD pair and then by acquiring an additional pair of images with a conventional phase stepping method to obtain en face OCT imaging at video rate. In a two-beam interferometer, the detected signal $S$ can be written as

$$
S=I_{R}+I_{S}+2 \sqrt{I_{R} I_{S}} \cos \phi,
$$

where $I_{R}$ and $I_{S}$ are the intensities of reference and sample beams, and $\phi$ is their relative phase at the detector. If this relative phase can be shifted by a well-controlled amount from its initial value, both $I_{S}$ and $\phi$ can be retrieved from a set of signals measured at well-defined phase shifts. The conceptually simplest way to do this is to take four interferograms $S_{n}$ with relative phase shifts equal to $n(\pi / 2)$, where $n=0,1,2,3$ corresponding to the mechanical motion of the reference mirror by $n(\lambda / 8)$. For the interference term, a straightforward calculation yields

$$
\begin{gathered}
S_{\text {int }}=16 I_{R} I_{S}=\left(S_{0}-S_{2}\right)^{2}+\left(S_{1}-S_{3}\right)^{2}, \\
\phi=\arctan \left(\frac{S_{1}-S_{3}}{S_{0}-S_{2}}\right)(\bmod \pi) .
\end{gathered}
$$

In principle, (5) is valid for monochromatic light. Whitelight interferograms can be described similarly with $I_{R}$ kept constant (independent of $\phi$ ), while $I_{S}$ is now a temporal envelope determined by the light temporal coherence. The phase $\phi$ is that of the intensity-modulated optical carrier frequency. In FF-OCT, the envelope quantity $S_{\text {int }}$, which is defined above is the one actually measured and which is proportional to $I_{S}$.

However, in FF-OCT, when the phase shift is induced by a mechanical displacement, the operation speed is limited by inertia. This technique is generally limited to the study of static objects in a mechanically stable environment due to its long measurement times. In the so-called "integratingbucket" technique, the intensity is integrated, while the phase is continuously shifted. The phase is usually shifted linearly in a sawtooth-like manner, and several integrated intensity values, or "buckets," are recorded. Compared to phase-stepping, this technique permits faster operation. The modulation is achieved by making the reference mirror oscillate. Unlike a sawtooth-like oscillation, which becomes distorted at high frequency, a sinusoidal oscillation remains steady, permitting a higher operational speed. There have been different optical schemes implemented for improving the speed of FF-OCT. Using optical Hilbert transform, Sato et al. reported a quadrature fringes wide-field (WF)OCT to realize the four-step phase-shifting method with
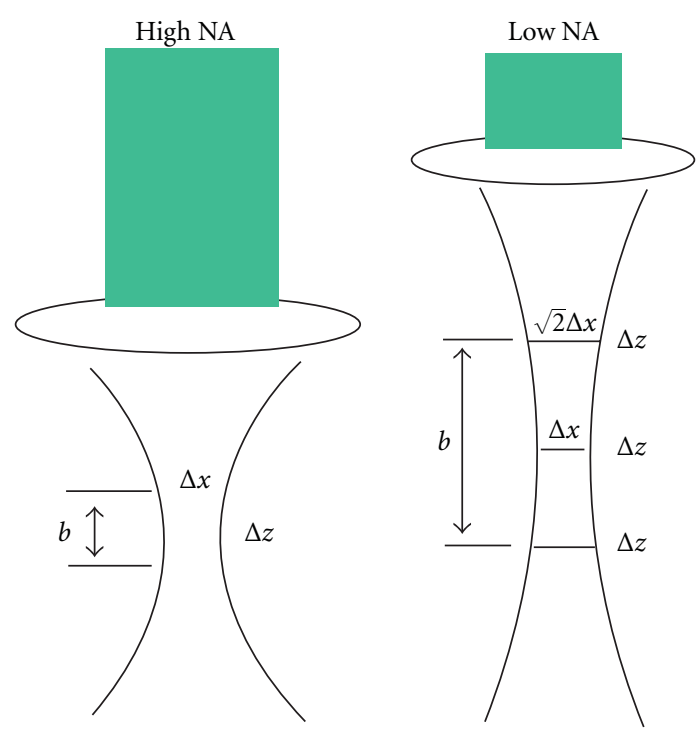

Figure 3: Relationship between focus spot size and depth of field for objective lens with high and low numerical aperture. The numerical aperture of an objective is defined as the refractive index of the lens immersion medium $n$ times the sine of the half-aperture angle $\alpha$ $(\mathrm{N} . \mathrm{A}=\sin \alpha)$.

a single InGaAs camera [42]. The system can reconstruct OCT images $(160 \times 160$ pixels $)$ at a frame rate of $30 \mathrm{fps}$ with a sensitivity of $90 \mathrm{~dB}$. 3D imaging of $4 \times 4 \times 2.9 \mathrm{~mm}^{3}$ was performed for the in situ rat brain using $1.3 \mu \mathrm{m}$ superluminescent diode (SLD) as a light source. Grieve et al. have reported FF-OCT with an acquisition speed of 250 fps by subtraction between two sequentially captured interference images [43]. The sensitivity was $71 \mathrm{~dB}$ with $4 \times$ 4 pixel binning. By averaging 10 images, 3D imaging $(320 \times$ $256 \times 160$ voxel) of the in vivo rat anterior segment was performed with an acquisition time of $6.4 \mathrm{~s}$. More recently, Watanabe and Sato. demonstrated a WF-OCT using CMOS camera, which can perform 3D en face imaging of size $2.6 \times$ $2.6 \times 1.2 \mathrm{~mm}^{3}(512 \times 512 \times 375$ voxel $)$ with a speed of $1500 \mathrm{fps}$ by calculating the absolute differences of two sequential images [44]. The sensitivity was $73 \mathrm{~dB}$ with $5 \times$ 5 pixel binning. The 3D OCT images of in vivo human finger were measured with 4 volume/s.

The main issue with conventional temporal phase stepping is the degradation of measurement speed of OCT image compared to that of imaging speed of CCD, this is because the reconstruction of one en face OCT image needs 3 or 4 interferograms depending upon the PSI algorithm used. Moreover, another issue with FF-OCT based on conventional phase stepping technique is that the sample should be stationary throughout the measurement time. Any movement of sample during measurement can directly affect the quality of the reconstructed en face OCT image. This significantly compromises the ability to image dynamic samples.

3.4. Sensitivity. The sensitivity or the optical dynamic range of an OCT system can be defined as the ratio of the signal 
power generated by a perfectly reflecting mirror to the noise of the system and is given by the equation:

$$
\mathrm{SNR}=\frac{I_{S}^{2}}{I_{N}^{2}},
$$

where $I_{S}$ is the photocurrent at the detector due to the interference term (4) and which in the case of an ideal $50: 50$ beam splitter and perfectly reflecting sample is equal to the total source power $P$. Therefore, the signal photocurrent can be expressed as

$$
I_{S}=\frac{\eta q_{e}}{h \nu_{0}} P,
$$

where $\eta$ the quantum efficiency of the detector, $q_{e}$ the electron charge, $\omega_{0}$ is the source center angular frequency, $\hbar$ is the Planck's constant divided by $2 \pi$, and $P$ is the integral of the source power spectral density. $I_{N}$ corresponds to the overall noise of the optical detection system and which has three significant sources: shot noise $\left(I_{\mathrm{SH}}\right)$, optical intensity noise or relative intensity noise $\left(I_{\mathrm{RIN}}\right)$, and the thermal noise $\left(I_{\mathrm{TH}}\right)$. Therefore, the total noise current is the sum of squares of each of the noise contributions and can be expressed as

$$
I_{N}^{2}=I_{\mathrm{SH}}^{2}+I_{\mathrm{RIN}}^{2}+I_{\mathrm{TH}}^{2}
$$

However, in the OCT system the optical detection scheme employed is heterodyne. Therefore, it can be arranged to achieve shot-noise-limited optical detection where by the reference arm optical field is much higher than that in the sample arm. This is often the case in OCT when imaging highly scattering and absorbing biological specimens. In shot-noise-limited case, the relative intensity noise. and the thermal noise become negligible. So, the total noise can be expressed as

$$
I_{N}=I_{\mathrm{SH}}=\sqrt{2 q_{e} I_{\mathrm{DC}} B},
$$

where $I_{\mathrm{SH}}$ is the rms shot noise, $B$ is the detection bandwidth, and $I_{\mathrm{DC}}$ is the DC photo current and is assumed to be entirely due to the reference arm's optical power. In case of shot noise limit, the overall noise variance can be expressed as

$$
I_{N}=\sqrt{\frac{\eta q_{e}}{h \nu_{0}} 2 P B} .
$$

Therefore, by substituting (7) and (10) into (6) yields

$$
\mathrm{SNR}=\frac{\eta P}{2 h \nu_{0} B} .
$$

Generally, the SNR is quoted as a figure of merit in logarithmic unit and is related to the power SNR by

$$
\mathrm{SNR}=10 \log _{10}\left(\frac{\eta P}{2 h \nu_{0} B}\right) .
$$

For a single detector, the measurement bandwidth $B=1 / \tau$ is a function of the acquisition time $\tau$ of the detector. Therefore, this expression implies that to increase either image acquisition speed or image resolution, which increase the measurement bandwidth, higher-source power is required to maintain the SNR performance of the system.

3.5. Merits and Demerits of FF-OCT. As compared to conventional point-scan OCT, FF-OCT can allow simplification of the optical setup as well as high imaging speed. FF-OCT can acquire a two-dimensional image at one depth over a transverse scan (en face image). Although single-point raster scanning systems can achieve video-rate acquisition, they have either limited sensitivity or a limited space-bandwidth product. En face OCT imaging can provide information, which may complement that provided by the longitudinal cross-sectional image with conventional point-scan OCT. En face OCT imaging is a very useful technique for the studies of eye compared to confocal scanning laser ophthalmoscopes and fundus cameras, which provide transversal images. It may also be suitable for the skin, which has transverse patterns of collagen.

For Poisson noise statistics, the variance of a random variable equals the mean, the signal to noise ratio (SNR) of a photo-detector signal should be proportional to the reciprocal square root of the number of photo-electrons [34]. Therefore, provided CCD array has the same quantum efficiency as a PIN diode, and there is sufficient light power, the SNR of two-dimensional array detectors can roughly be $N$ times larger than that of a single-element photodetector where $N$ is the number photo cells of an array detector. However, to have comparable number of photons detected per detector cell as in single-point raster scanning systems, FF-OCT systems require roughly $N$ times larger light power. Moreover, FF OCT allows the possibility to use inexpensive thermal source by taking the advantage of high axial resolution.

However, to take advantage of a full-field detecting scheme, several challenges are recognized: one is optical crosstalk the between detecting pixels at samples, which is described in the above section and second is speckle noise. These two obstacles have similar origin that is spatial and temporal coherence of illuminating light. Speckle is a major obstacle in measuring specific structural properties (e.g., birefringence) $[13,45-47]$ of tissue specimens and observing structured features that approach the diffraction limit [48]. Although origin of speckle in OCT images is still being investigated [49], a common view is that the effect originates in distorted wavefronts of light returning from tissue that result in constructive and destructive features in the interferogram. The third obstacle relies on the relatively slow frame rate and poor sensitivity of the commercially available CCD. In FF-OCT, the direct use of CCD cameras for the heterodyne detection is limited by its relatively slow frame rate $[27,50]$. Moreover, the strong optical DC intensity from the reference arm degraded the dynamic range available for the detection of AC interferometric component. Therefore, for extracting the interferometric component FF-OCT utilizes homodyne detection technique such us phase stepping interferometric method [37]. However, the poor dynamic range of available CCD is a major limiting factor for imaging highly scattering specimens. 


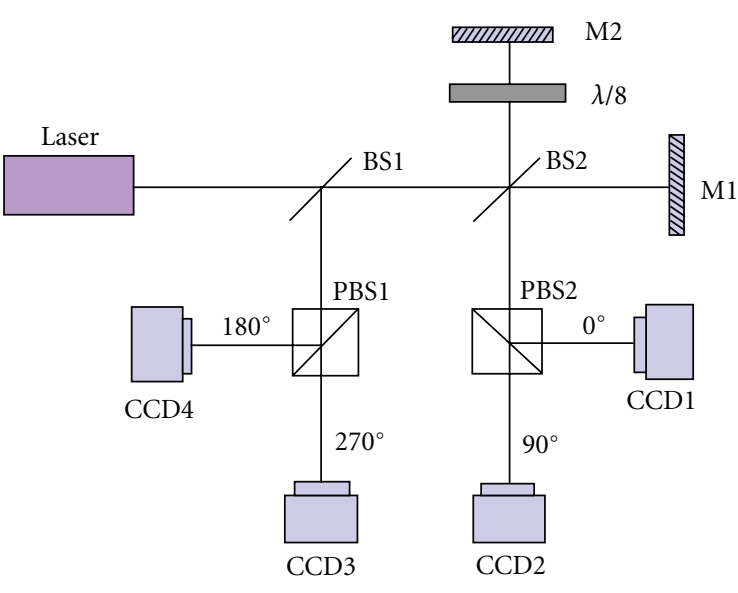

(a)

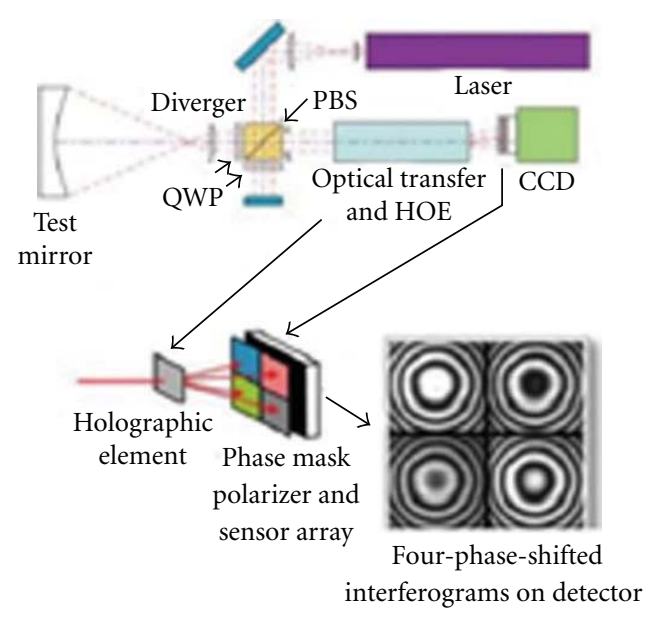

(b)

FiguRE 4: (a) simultaneous phase-shifting interferometer based on four cameras; (b) simultaneous phase-shifting interferometer based on single camera (phase Cam-from, [31]).

\section{Single-Shot FF-OCT}

Single-shot full-field OCT (SS-FF-OCT) is relatively a new concept, which aims to improve the imaging speed of conventional FF-OCT by simultaneous acquisition of 3 or 4 interferograms on a single- or multi- charge-coupled device (CCD) camera $[32,51,52]$. The basic principle of SS FFOCT is very similar to the simultaneous phase-shifting interferometric method $[31,53]$ which have been developed over the years for high-speed vibration insensitive optical testing.

4.1. Single-Shot Phase-Shifting Interferometry. Optical interferometric schemes can allow a variety of surface measurements from optical elements such as mirrors to highly scattering biological specimens with very high spatial resolution. Numerous types of interferometric techniques have been reported using many kinds of light sources coupled to the interferometer and the way to analyze the interference patterns $[54,55]$. Laser-based phase-shifting interferometry (PSI) can perform three-dimensional (3D) surface measurements with subnanometer resolution by analysis of a few sequences of phase-shifted monochromatic interferograms. PSI can be accomplished either by sequentially introducing a temporal phase-shifting method or by spatially splitting the beam into parallel channels for simultaneous phaseshifting method. In conventional temporal phase-shifting interferometry, the interferometers have an element to introduce three or more known phase shifts in the path of the reference light. Measuring the interference image at each of these phase shifts and by analyzing, the phase distribution of signal lights can be quantitatively calculated. However, the interferometer using temporal phase-shifting method is very sensitive to vibration because the various phase-shifted frames of interferometric data are taken at different times, and vibration causes the phase shift between the data frames to be different from the desired value. Instead, in simultaneous phase-shifting interferometry, the spatially phase-shifted three or four interferograms were simultaneously acquired in a time several order of magnitude less than temporal phase shifting, thus eliminates the measurement errors caused by the sample vibrations or movements.

Several types of simultaneous phase-shifting method have been developed over the years, and these techniques utilize conventional beam splitters and polarization optics to produce three or four phase shifted images on a singleor multi- charge-coupled device (CCD) for simultaneous acquisition [56-59]. Figure 4 shows the basic schemes of simultaneous interferometry. Most of these methods require relatively complex optical and electronic arrangements and have had limited practical applications. Recently, novel simultaneous PSIs that use diffractive elements to simultaneously image three or more interferograms onto a single-CCD sensor have been reported by several authors [60-63]. These techniques are considerably more compact and less expensive compare to the multicamera arrangement. However, the diffractive elements are available only over a small spectral band due to dispersion and chromatic distortion inherent in their design. Thus, they are not capable of working with white light or short-coherence length source interferometers [31]. More recently, single-shot PSI based on pixelated phase mask was introduced in which micropolarizers were used to spatially multiplex the phase-shifted interferograms $[64,65]$ and which works well over a large wavelength band. However, such type of phase mask is difficult to manufacture accurately and needs highly sophisticated technologies [31].

\subsection{Single-Shot Phase-Stepped Wide-Field Coherence-Gated Imaging. The first single-shot approach in FF-OCT for depth-resolved imaging was demonstrated by C. Dunsby et al. in 2003 [32]. The schematic of the experimental setup is shown in Figure 5. The experimental setup is based on a polarizing Michelson-based interferometer followed by a four-channel phase stepper optics. An LED was used as a light source, and at the output of the interferometer both}




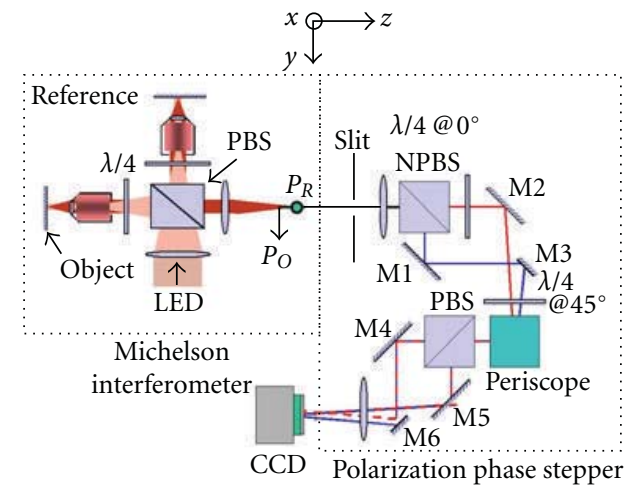

FIGURE 5: Single-shot wide-field coherence-gated imaging setup proposed by Dunsby et al. (from [32]).

the signal and reference light are polarized orthogonally. The fast axis of QWP1 is parallel to $y$-axis. That of QWP2 is at $45^{\circ}$ with respect to $z$-axis. The linearly polarized lights were changed into the circularly polarized lights and were split into four interference images with orthogonal components. The four retardations of $0^{\circ}, 270^{\circ}$ and $180^{\circ}, 90^{\circ}$ are made from Beam1 and Beam2, respectively. Periscope was used to rotate beams by an angle of $90^{\circ}$ for the simplification of optical alignment. Thus, the four-phase-stepped interference images can simultaneously capture using a single-CCD camera. The temporal resolution of the system depends only on the frame rate of the CCD camera. Depth-resolved images of a moving watch cogs with a field of view of $3.6 \times 2.6 \mathrm{~mm}$ $(276 \times 196$ pixels $)$ has been demonstrated as dynamic a sample. Those images of an onion slice with a dimension of $0.27 \times 0.25 \times 0.21 \mathrm{~mm}^{3}$ were also shown as biological specimen. The images were acquired at a speed of $16.5 \mathrm{fps}$ with $2 \times 2$ pixel binning to obtain the signal noise ratio of $44 \mathrm{~dB}$.

However, the optical configuration of this four-channel phase-stepper optics was quite complex and which limits this system's performance for practical implementation. Any differences caused by image projection, optical aberration, and response uniformity will directly influence the image reconstruction accuracy. Moreover, for this proposed system design, a couple of corrections parameters were required to successfully reconstruct the image. All these correction parameters were retrieved from a set of calibration images, which were determined manually during the system characterization.

\section{Compact Single-Shot FF-OCT}

We have developed a compact single-shot full-field OCT technique [52] based on a Linnik type polarization Michelson interferometer and a four-quadrature phase-stepper, which can simultaneously capture four-quadrature phasestepped interferograms on a single CCD. Main advantages of this phase-stepper optics are its compactness, reduced distortions, and reduced imbalances of magnifications and intensities due to the equal optical path lengths and symmetric design of the four channels. Eventually the reconstruction algorithm becomes simple, and the sensitivity could be improved. The four-phase-stepping algorithm includes only four parameters to correct small-intensity imbalances among the four channels. Those parameters are calculated and corrections for each subimage were performed automatically in real time. Using a superluminescent diode as a light source with center wavelength of $842 \mathrm{~nm}$ and spectral width of $16.2 \mathrm{~nm}$, the system yields an axial resolution of $19.8 \mu \mathrm{m}$ and covers a field of view of $280 \times 320 \mu \mathrm{m}^{2}(220 \times$ 250 pixels) with a transverse resolution of $4.4 \mu \mathrm{m}$ by using a 10x microscope objective ( $0.3 \mathrm{NA}$ ). Three-dimensional OCT images of biological samples such as an onion slice and a diaptomus were obtained without any image averaging or pixel binning. In addition, in vivo depth-resolved dynamic imaging capability of the system was demonstrated by showing the beating internal structure of a diaptomus with a fame rate of $5 \mathrm{fps}$.

\subsection{Optical Scheme}

5.1.1. Polarizing Interferometer. The experimental setup consists of a bulk optics-based polarizing Michelson interferometer with Linnik configuration and a four-quadrature phasestepper optics as illustrated in Figure 6. A SLD (Anritsu, GS8C500ALY) emits a beam with Gaussian spectrum of central wavelength at $842 \mathrm{~nm}$ and a spectral bandwidth of $16.2 \mathrm{~nm}$ (FWHM). The lens L1 (focal length $12 \mathrm{~mm}$ ) collimates the light from the SLD. Using the half-wave plate1 (HWP-1) and polarizer (P-1), the light is linearly polarized at $+45^{\circ}$ and coupled into the polarizing interferometer. The 2D interferometer consists of a polarizing beam splitter (PBS), which splits the incoming light into two orthogonally polarized lights, two-quarter wave plates (QWP-1 and QWP2 ), and objective lenses. After the PBS, the two orthogonally polarized lights pass the quarter wave plates placed at the sample and reference arms. Two identical microscope objectives are utilized in both reference and sample arms. After round-trip transmission through wave plates, the polarization states of lights in sample and reference arms are exchanged. At the output of the interferometer the reference light is linearly polarized along $x$-axis ( $x$-polarization) and the sample light is linearly polarized along $y$-axis $(y$ polarization).

5.1.2. Four-Channel Phase-Stepper Optics. The detailed schematic of the four-channel phase-stepper optics is shown in Figure 7. It consists of a half-wave plate (HWP-2), a rectangular slit (S), two lenses (L2 and L3), a dual-channel parallel beam splitter (DCPBS), a quarter-wave plate (QWP3), a compensation glass plate (CGP), and a Wollaston prism (WP). The half-wave plate with its fast axis oriented at $22.5^{\circ}$ to the $x$-axis rotates the polarization states of the reference (vertical polarization) and sample arm light (horizontal polarization) with an angle of $45^{\circ}$. The use of HWP-2 could be eliminated by tilting the phase-stepper optics and CCD camera to an angle of $45^{\circ}$. The dual-channel parallel beam 


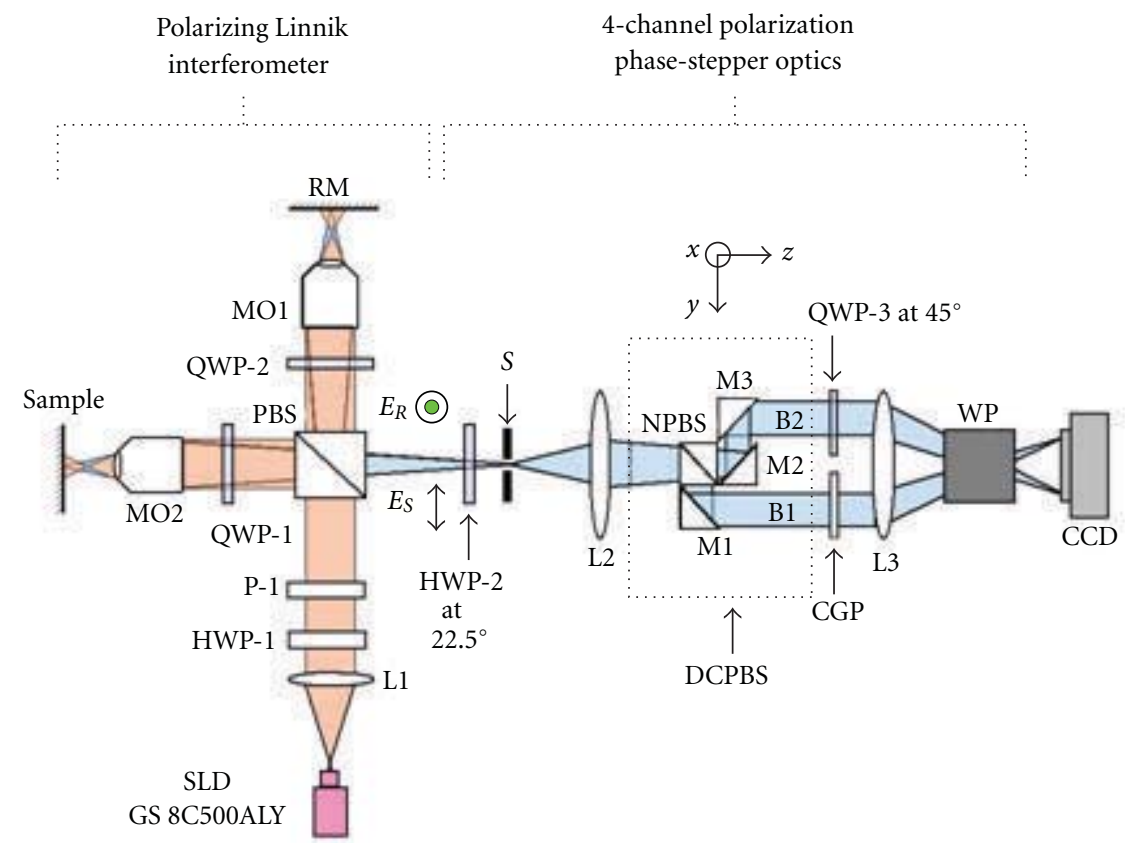

Figure 6: Experimental setup (lateral view); L1-3 lenses, HWP: half-wave plate, P: polarizer, PBS: polarizing beam splitter, QWP: quarterwave plate, MO1-2: microscopic objectives $(0.3 \mathrm{NA} / 10 \times$ or $0.25 \mathrm{NA} / 10 \times$ or $0.1 \mathrm{NA} / 5 \times)$, RM: reference mirror, S: rectangular slit, NPBS: nonpolarizing beam splitter, M1-3: mirrors, CGP: compensation glass plate, WP: wollaston Prism, and CCD: charge-coupled device.

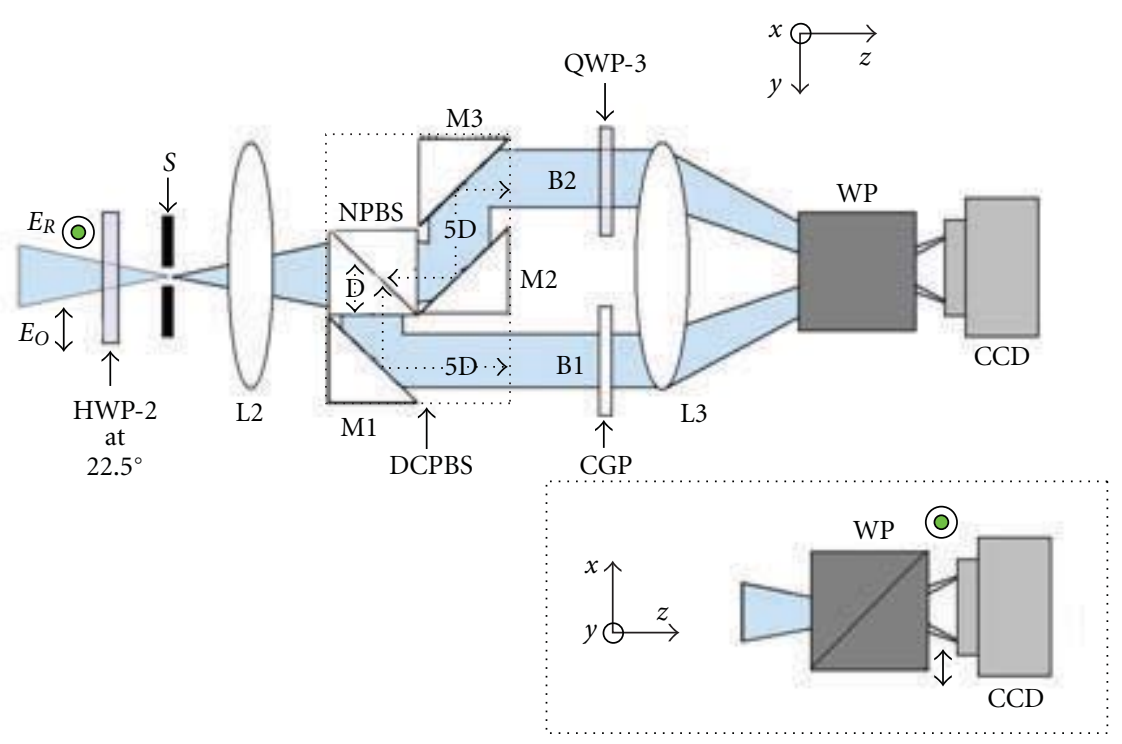

FIGURE 7: Schematics of the four-quadrature phase-stepper (lateral view); $E_{S}$ : signal light, $E_{R}$ : reference light, HWP: half-wave plate, L2-3 Lenses, NPBS: nonpolarizing beam splitter, M1-3: mirrors, QWP: quarter wave plate, CGP: compensation glass plate, WP: wollaston prism, $\mathrm{D}(5 \mathrm{~mm})$ corresponds to the half-size of NPBS. Inset shows the separation of incident beam into two orthogonally polarized images by the Wollaston.

splitter is constructed using a nonpolarizing beam splitter (NPBS, $10 \times 10 \times 10 \mathrm{~mm}^{3}$ ) and three gold-coated mirrors (M1-M3) to split the incident beam from the interferometer into two parallel beams with the equal optical path length. As shown in Figure 7, D corresponds to the half-size length of the NPBS, and the optical path length introduced by the DCPBS was 5D for both Beam 1 and Beam 2, which is indicated by the bold dotted line in Figure 7. Beam 2 then incident into QWP-3 with its fast axis oriented at an angle of $45^{\circ}$ to the $x$-axis. In order to compensate the optical path 
difference due to QWP-3, a compensation glass plate (CGP) with same thickness of QWP-3 was inserted into the optical path of Beam 1. Then, the two beams were launched into a Wollaston prism with a separation angle of $4.8^{\circ}$ (aperture $15 \times 15 \mathrm{~mm}^{2}$ ), which splits the incident beam into two orthogonal components of s- and p-polarization. The inset in Figure 7 shows the separation of the incident beam into two orthogonally polarized components.

The real image formed at the plane of the rectangular slit $S$ is relayed to the CCD plane by a pair of lenses L2 $(125 \mathrm{~mm})$ and $\mathrm{L} 3(75 \mathrm{~mm})$. The role of $\mathrm{L} 3$ is to reduce the real image area formed by L2 to fit into the CCD imaging area. The measured magnification of the entire optics was 7.7, which has almost good agreement with the calculated magnification 7.5. The entire dimension of this phasestepper is axial length (L2 to CCD front face) of $300 \mathrm{~mm} \times$ width of $40 \mathrm{~mm}$. Eventually four-quadrature phase-stepped interference images can be simultaneously obtained through this phase-stepper optics. The polarization states of light were analyzed to calculate the intensities of four interference images using Jones vectors $J$ and matrix $T$ [66]. The electric fields of the signal and reference lights that enter the phasestepper can be expressed as:

signal light:

$$
E_{S}=E_{S 0} e^{j \varphi_{S}}\left[\begin{array}{l}
0 \\
1
\end{array}\right]=E_{S 0} e^{j \varphi_{S}} J_{S}
$$

reference light:

$$
E_{R}=E_{R 0} e^{j \varphi_{R}}\left[\begin{array}{l}
1 \\
0
\end{array}\right]=E_{R 0} e^{j \varphi_{R}} J_{R},
$$

where $E_{S 0}, E_{R 0}, \phi_{S}$, and $\phi_{R}$ are the amplitudes and phases of signal and reference lights, respectively.

Passing through the lens L3, the electric fields of the signal and reference lights for Beam 1 and Beam 2 are given by

$$
\begin{aligned}
& E_{1 S}=E_{S 0} e^{j \phi_{S}} T_{M} T_{R} T_{\mathrm{HWP}} J_{S}=E_{S 0} e^{j \phi_{S}} \frac{1}{2}\left[\begin{array}{c}
1 \\
-1
\end{array}\right] \\
& E_{1 R}=E_{R 0} e^{j \phi_{R}} T_{M} T_{R} T_{\mathrm{HWP}} J_{R}=E_{R 0} e^{j \phi_{R}} \frac{1}{2}\left[\begin{array}{c}
1 \\
1
\end{array}\right] \\
& E_{2 S}=E_{S 0} e^{j \phi_{S}} T_{\mathrm{QWP}} T_{M} T_{M} T_{T} T_{\mathrm{HWP}} J_{S}=E_{S 0} e^{j \phi_{S}} \frac{j}{2}\left[\begin{array}{c}
-1 \\
1
\end{array}\right] \\
& E_{2 R}=E_{R 0} e^{j \phi_{R}} T_{\mathrm{QWP}} T_{M} T_{M} T_{T} T_{\mathrm{HWP}} J_{R}=E_{R 0} e^{j \phi_{R}} \frac{1}{2}\left[\begin{array}{l}
1 \\
1
\end{array}\right]
\end{aligned}
$$

where $T_{M}, T_{R}, T_{T}, T_{\mathrm{QWP}}$, and $T_{\mathrm{HWP}}$ are the Jones matrixes for the reflection of mirror, the reflection of beam splitter, the transmission of beam splitter, and QWP (fast axis at $45^{\circ}$ to the $x$-direction) and HWP (fast axis at $22.5^{\circ}$ to the $x$ direction), respectively, which can be expressed by assuming that the conductivity of metals in mirrors is infinity [67] as follows

$$
\begin{gathered}
T_{M}=\left[\begin{array}{cc}
-1 & 0 \\
0 & 1
\end{array}\right] ; \quad T_{R}=\frac{1}{\sqrt{2}}\left[\begin{array}{cc}
-1 & 0 \\
0 & 1
\end{array}\right] ; \quad T_{T}=\frac{1}{\sqrt{2}}\left[\begin{array}{ll}
1 & 0 \\
0 & 1
\end{array}\right], \\
T_{\mathrm{QWP}}=\frac{1}{2}\left[\begin{array}{cc}
1-j & 1+j \\
1+j & 1-j
\end{array}\right] ; \quad T_{\mathrm{HWP}}=\frac{1}{\sqrt{2}}\left[\begin{array}{cc}
1 & 1 \\
1 & -1
\end{array}\right] .
\end{gathered}
$$

Through the Wollaston prism, the resulting intensities $I$ for each interference image of A-D can be obtained as follows:

$$
\begin{aligned}
& \mathrm{A}: I_{1 X}=\frac{1}{4}\left(E_{S 0}^{2}+E_{R 0}^{2}+2 E_{S 0} E_{R 0} \cos \left(\varphi_{S}-\varphi_{S}\right)\right), \\
& \mathrm{B}: I_{1 Y}=\frac{1}{4}\left(E_{S 0}^{2}+E_{R 0}^{2}+2 E_{S 0} E_{R 0} \cos \left(\varphi_{S}-\varphi_{R}+180^{\circ}\right)\right), \\
& \mathrm{C}: I_{2 X}=\frac{1}{4}\left(E_{S 0}^{2}+E_{R 0}^{2}+2 E_{S 0} E_{R 0} \cos \left(\varphi_{S}-\varphi_{R}+270^{\circ}\right)\right), \\
& \mathrm{D}: I_{2 Y}=\frac{1}{4}\left(E_{S 0}^{2}+E_{R 0}^{2}+2 E_{S 0} E_{R 0} \cos \left(\varphi_{S}-\varphi_{R}+90^{\circ}\right)\right) .
\end{aligned}
$$

5.2. Image Acquisition and Reconstruction. The four-phasestepped interference images were captured using a 12 bit cooled progressive scan interline CCD with a frame rate of $28 \mathrm{fps}(656 \times 494$ pixels $)$ without any pixel binning and image averaging. With an external trigger mode to change the speed of image acquisition, the frame rate of the CCD was get reduced to $14 \mathrm{fps}$. The image is transferred from the camera to a personal computer (Intel P-4, 3.06 GHz) via an image acquisition card (IMAQ PCI 1422, National Instruments Corp., USA).

However, practically, there is an influence of darkcurrent noise and slight intensity unbalances among each of the four-phase-stepped images. In order to eliminate this problem, after the full frame capture the image is divided into four subimages, and then the constant dark-current noise component is subtracted from each of this subimages. Prior to this background subtraction, the four subimage positions have been decided in the full frame image of $656 \times 494$ pixels area. Then, for reconstructing the en face OCT image $I_{\mathrm{OCT}}$, we used the below (21), which is obtained by introducing four intensity calibration parameters to compensate intensity unbalances:

$$
I_{\mathrm{OCT}}=\left[\left(a I_{1 X}-b I_{1 Y}\right)^{2}+\left(c I_{2 X}-d I_{2 Y}\right)^{2}\right]^{1 / 2},
$$

where $a, b, c$, and $d$ are the intensity calibration parameters. These coefficients are calculated as the ratio of the smallest mean intensity among the four subimages to the mean intensity of each subimage; therefore, all the mean intensity of the subimages are equalized to the smallest mean intensity. A custom software developed in Lab VIEW-IMAQ-Vision domain calculates the intensity coefficients in real-time by means of the specific functions in IMAQ-Vision. The image acquisition, image processing of (21) and displays of en face images can be done with a frame rate of $28 \mathrm{fps}$ in realtime. The proposed system was capable of automatically compensate small-intensity unbalances among the four 


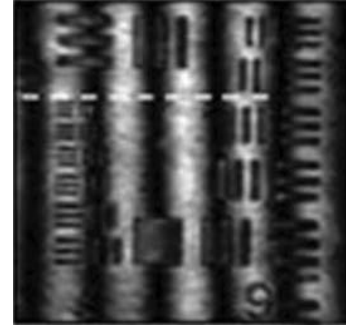

(B)

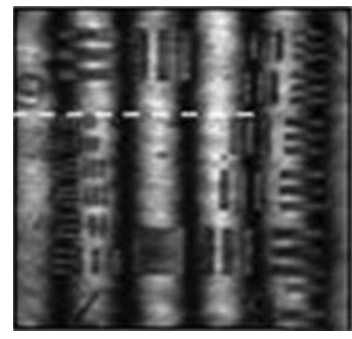

(A)

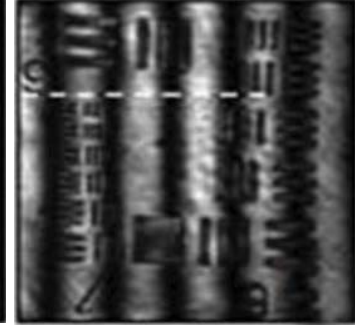

(D)

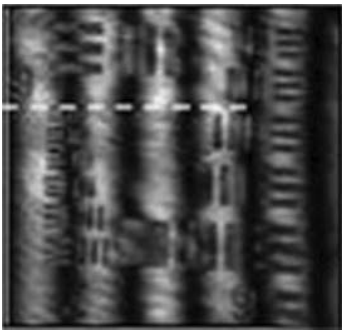

(C)

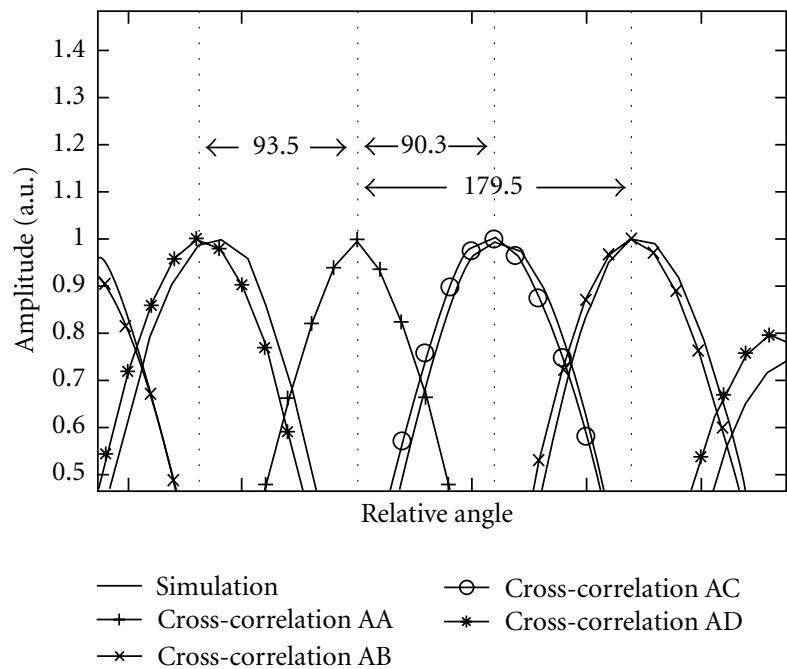

(b)

FIGURE 8: (a) Four-phase-stepped interference images captured on the CCD camera. Then, intensity profile is taken at places indicated by white dotted lines for cross-correlation. (b) Cross-correlation peaks of intensity profiles in images A, B, C, and D with that in image A. The peak separations give relative-stepped phases of each interference image. Simulated results of cross-correlations are shown with solid narrow lines.

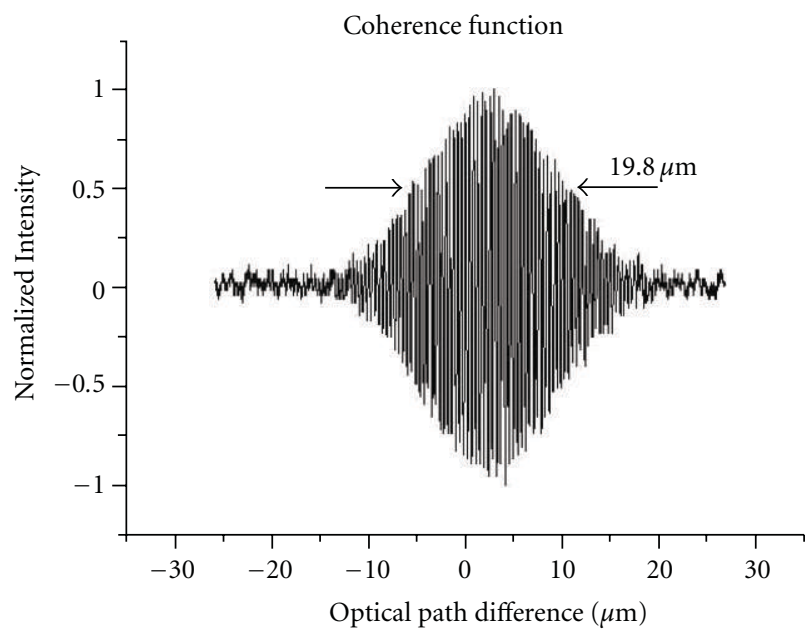

Figure 9: Coherence function measured.

subimages in real-time. Unlike the early version of singleshot OCT [32], this system does not need any retrievals to obtain correction parameters. For measuring $3 \mathrm{D}$ volumetric image, en face images were sequentially acquired by scanning the reference mirror along the optical axis with the constant step using a high-precision motorized translation stage.

\subsection{Basic Characteristic}

5.3.1. Stepped-Phase Measurement. Figure 8(a) shows the four-quadrature phase-stepped image of a USAF test target. Using the cross-correlation between two intensity profiles,

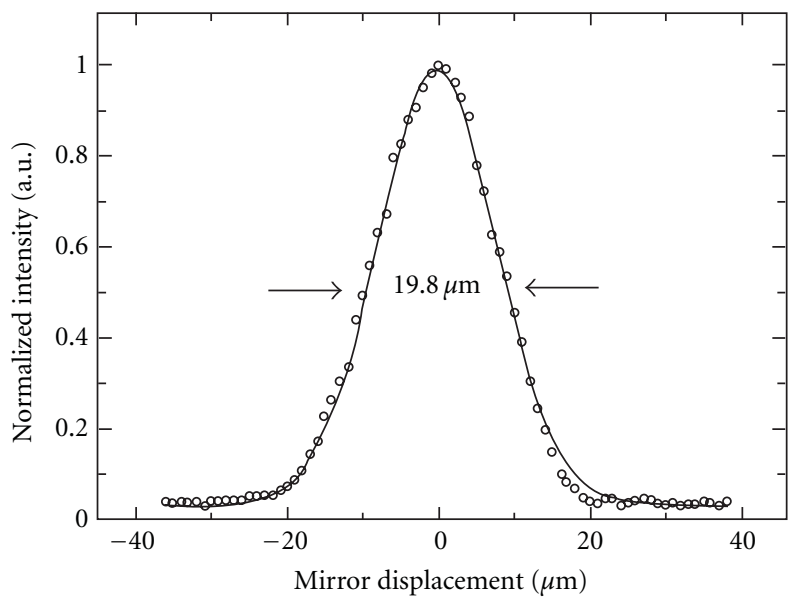

PSF curve Gauss fit

Figure 10: Measured axial point spread function (coherence function) by displacing the reference mirror, and the solid line represents the corresponding Gaussian fitting curve.

the relative stepped phases of image $\mathrm{B}$ to $\mathrm{D}$ with image A were measured at $-179.5^{\circ},-90.3^{\circ}$, and $93.5^{\circ}$, respectively as shown in Figure 8(b). Those measured phases were almost corresponding to the calculated values given by (20). The measured as well as the simulated intensity profiles were normalized prior to the cross-correlation calculation.

5.3.2. Axial Resolution and System Sensitivity. First, the axial point spread function was obtained by measuring 


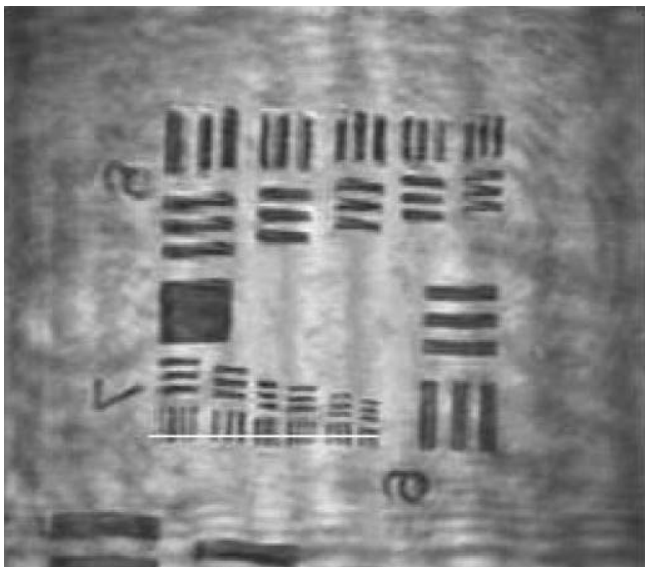

(a)

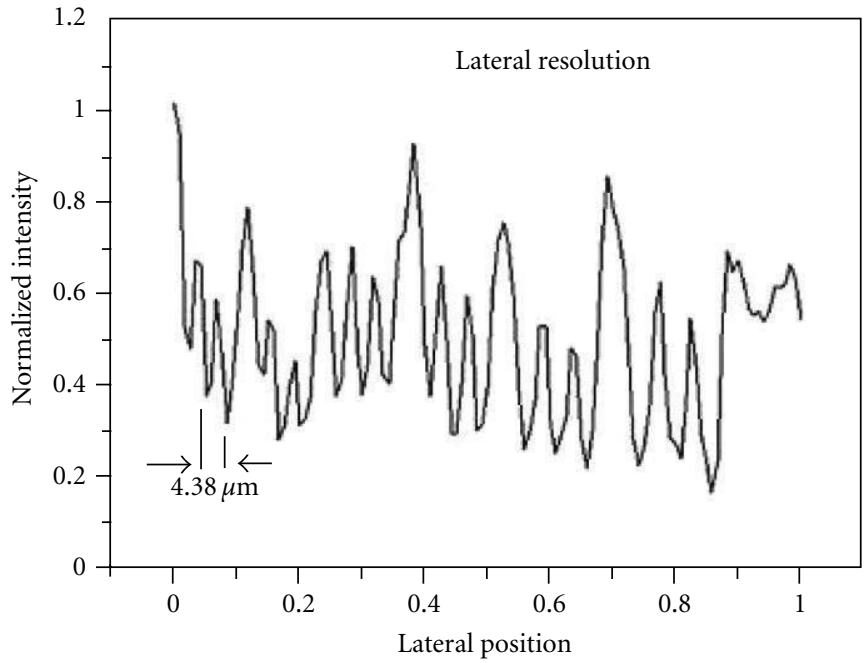

(b)

Figure 11: (a) reconstructed en face OCT image of a USAF 1951 test target $(220 \times 250$ pixels $)$ with a field of view of $280 \times 320 \mu \mathrm{m}^{2}$ using an objective of NA $0.25(10 \times)$ and (b) intensity profile of the 7th group elements taken at the place indicated by the white line in Figure $11(\mathrm{a})$.

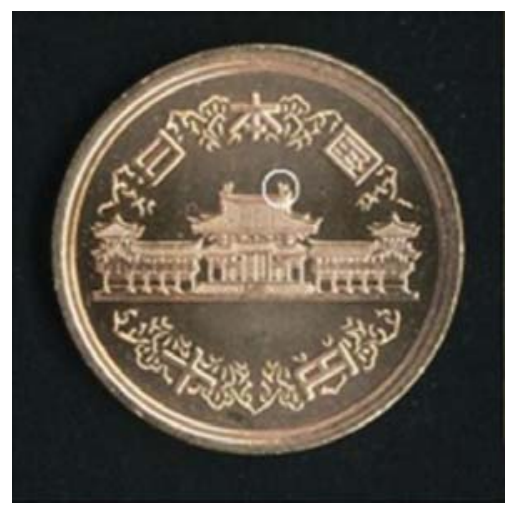

(a)

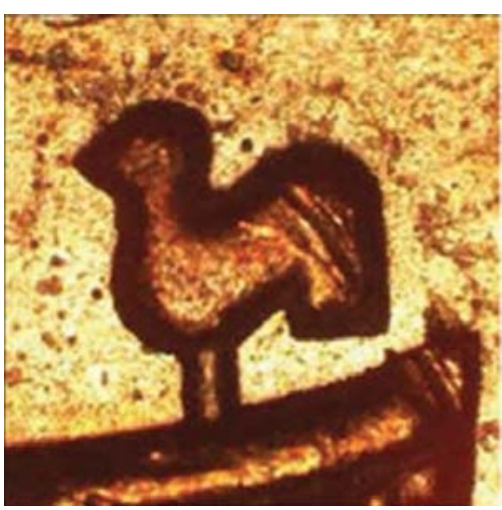

(b)

FIGURE 12: (a) 10-yen Japanese coin, white circle shows the bird imprint and (b) microscopic image of bird imprint.

the coherence function of the optical source measuring, which is shown in Figure 9, the measured value was $19.8 \mu \mathrm{m}$ (FWHM). Next, for measuring the actual axial resolution of the implemented system, OCT images were acquired by scanning the reference mirror in steps of $1 \mu \mathrm{m}$ with a highprecision motor stage by fixing the sample mirror at the focal point. Figure 10 shows that the obtained resolution was about $19.8 \mu \mathrm{m}$, which is in close agreement with the calculated value of $19.2 \mu \mathrm{m}$ using the central wavelength at $842 \mathrm{~nm}$ and a spectral bandwidth of $16.2 \mathrm{~nm}$ (FWHM).

The relations between the axial resolution, the numerical aperture (NA) of the objective, and the coherence length of optical source were reported elsewhere [26]. In the case of low-NA objectives $(\leq 0.4)$, the axial resolution is determined by the coherence length of the source, as in conventional OCT systems. However, in the case of high-NA objective $(\geq 0.4)$, the axial resolution is prescribed by the NA of the objective rather than by the coherence length of the source.
The objectives used in our experimental setup has low numerical aperture $(\leq 0.3)$, which is corresponds to one of the above-described condition.

The sensitivity of the system was measured by using a mirror as test object. During measurement, the irradiation power on the sample surface was around $250 \mu \mathrm{W}$, and the CCD exposure time set was $0.7 \mathrm{~ms}$. Inserting an OD-2 (optical density-2) filter in the sample arm attenuates the signal light with a roundtrip attenuation of $40 \mathrm{~dB}$. A variable attenuator was used in the reference arm to keep the CCD just close to the saturation level. Then, the measured overall sensitivity was approximately $-66.5 \mathrm{~dB}$. Under this condition, the reflectivity of reference mirror was measured at 3.0\%.

We estimated the theoretical sensitivity of the system by introducing the photon noise [26]. The signal intensity $S_{\text {INT }}$ of interference images is given by

$$
S_{\mathrm{INT}}=\left(R_{S}+R_{R}+R_{\mathrm{INC}}+2 \sqrt{R_{S} R_{R}} \cos \varphi\right) I_{0} \tau,
$$




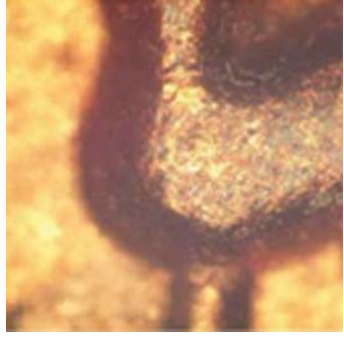

(a)

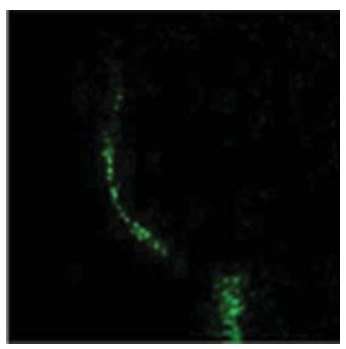

(c)

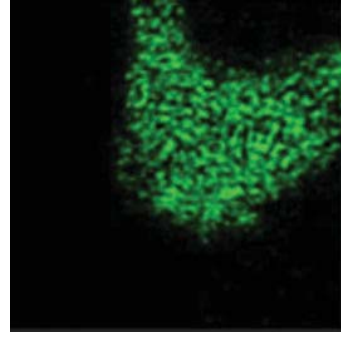

(b)

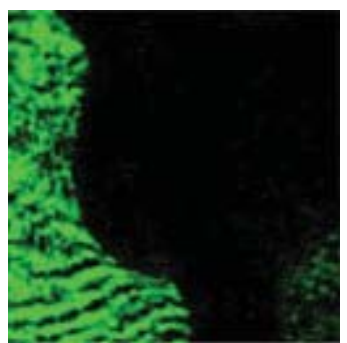

(d)
Figure 13: (a) Microscopic image of the bird on the 10-yen Japanese coin. (b)-(d) reconstructed en face OCT images of top, middle, and bottom surfaces of the bird imprint.

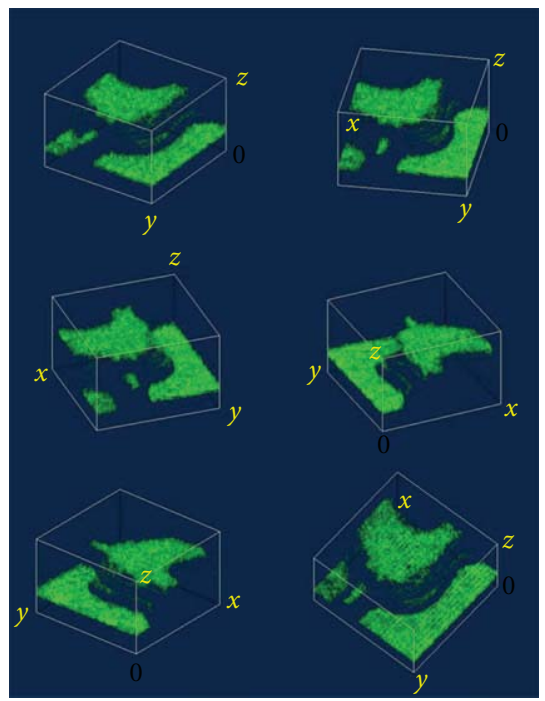

Figure 14: Reconstructed 3D volumetric image of the coin in different axis orientation $\left(425 \times 425 \times 160 \mu \mathrm{m}^{3}\right)$.

where $R_{S}$ is the internal reflectance of samples, $R_{R}$ is the reflectance of the reference mirror, $R_{\mathrm{INC}}$ represents the portion of incoherent light emitted from the sample, and $\phi$ is the phase difference between reference and signal lights. Assuming that the camera is operated nearby its saturation, the signal to noise ratio (SNR) can be written by

$$
\mathrm{SNR}=\frac{4 R_{R} R_{S} I_{0}^{2} \tau^{2}}{\xi}=\frac{\xi \gamma^{2}}{(1+\gamma)^{2}},
$$

where $\xi$ is the full-well capacity of CCD, and $\gamma$ is the fringe visibility. With conditions that the SNR of 2 is the minimum detectable limitation, and the reflectance of samples is much smaller than that of reference and incoherent light, the fringe visibility can be given by

$$
\gamma \cong \frac{2 \sqrt{R_{R} R_{S}}}{R_{R}+R_{\mathrm{INC}}}=\sqrt{\frac{2}{\xi}}
$$

The minimum detectable reflectivity $R_{S \min }$ is given by

$$
R_{S \min }=\frac{\left(R_{R}+R_{\mathrm{INC}}\right)^{2}}{2 \xi R_{R}} .
$$

Therefore, the sensitivity can be expressed by

$$
10 \log \left(R_{S \min }\right)=10 \log \frac{\left(R_{R}+R_{\mathrm{INC}}\right)^{2}}{2 \xi R_{R}} .
$$

The sensitivity was calculated at $-63 \mathrm{~dB}$ to $-65 \mathrm{~dB}$ with the measured reflectivity of $3.0 \%$ in the reference arm and the full-well capacity from 30,000 to 50,000 according to the CCD specification. Since the mirror was used as an object, the reflectivity of incoherent light was neglected. The calculated sensitivity almost corresponds to the measured one.

5.3.3. Lateral Resolution. The lateral resolution is determined by the NA of the microscopic objectives and the wavelength. For obtaining the en face images, we employed three sets objectives with NA of $0.25(10 \times), 0.3(10 \times)$, and $0.1(5 \times)$. For the evaluation of lateral resolution, we measured the OCT image of a standard USAF test target using an objective of NA $0.3(10 \times)$. The Measured OCT images with a field of view of $280 \times 320 \mu \mathrm{m}^{2}$ is shown in Figure $11(\mathrm{a})$. We could resolve the 6 th elements in 7 th group as shown in Figure 11(b). The lateral resolution was measured at $4.4 \mu \mathrm{m}$, which was almost corresponds to $3.4 \mu \mathrm{m}$ calculated by $(1.22$ $\left.\times \lambda_{0}\right)$ /NA. Similarly, for objectives with NA $0.1(5 \times)$ and NA $0.25(10 \times)$ the measured resolutions were $11 \mu \mathrm{m}$ (calculated $10.2 \mu \mathrm{m}$ ) and $4.4 \mu \mathrm{m}$ (calculated $4.1 \mu \mathrm{m}$ ), respectively.

\subsection{Measurement Result}

5.4.1. 3D Imaging of a Rough Metallic Surface. To demonstrate the imaging capability of the system, first we captured the depth resolved en face OCT images of a bird imprint on a 10-yen Japanese coin, which is shown in Figure 12(a). The microscopic image of the imprint on the coin under white light illumination is shown in Figure 12(b). The coin with rough surfaces is made of bronze $(70 \% \mathrm{Cu}$ and $30 \% \mathrm{Zn})$. During measurement, the microscopic objective used was $5 \times$ with NA of 0.1 . The irradiated power and the exposure time were $250 \mu \mathrm{W}$ and $0.7 \mathrm{~ms}$, respectively. During measurement, the sample was fixed under the objective lens and the en face OCT images were acquired by scanning the reference mirror with a $1 \mu \mathrm{m}$ step up to the depth of $160 \mu \mathrm{m}$. Using the equation of $\pi \Delta x^{2} /(2 \lambda)$, the depth of focus (DF) calculated was $90 \mu \mathrm{m}$ with the measured lateral resolution. Therefore, we could observe the degradation of image quality, which is caused by the mismatch of coherence-gated plane and the 


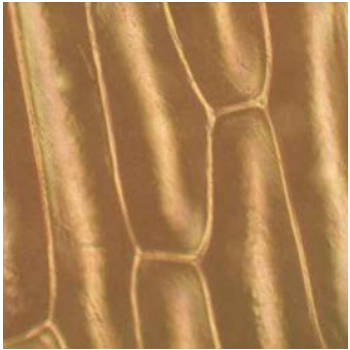

(a)

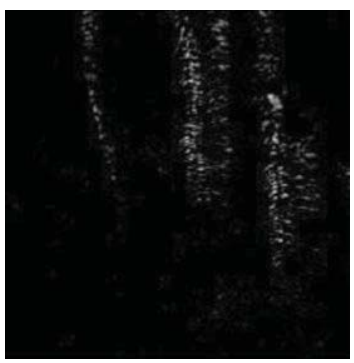

(e)

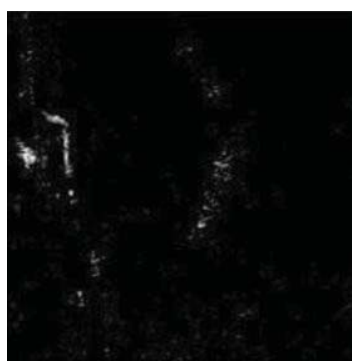

(i)

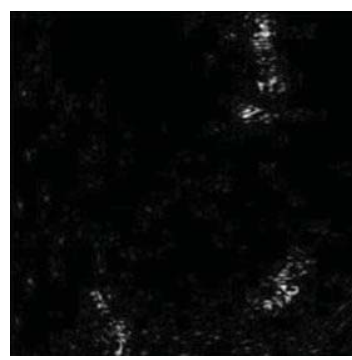

(m)

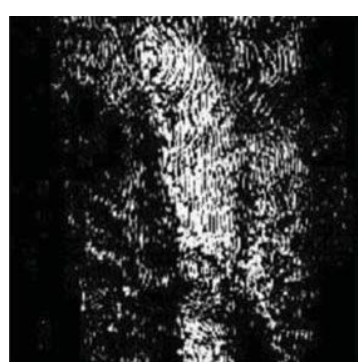

(b)

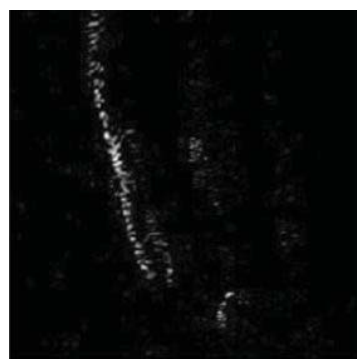

(f)

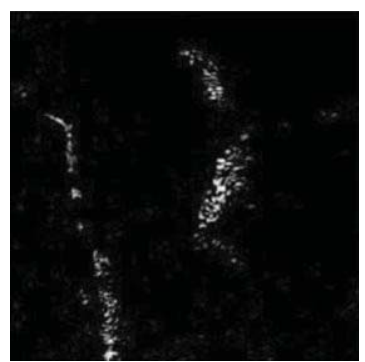

(j)

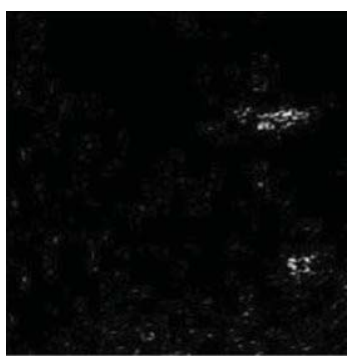

(n)

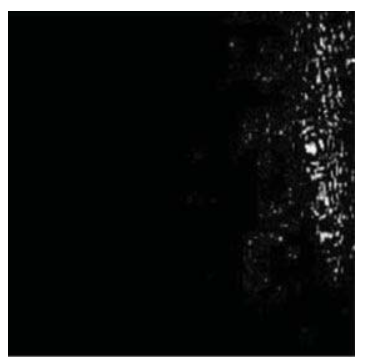

(c)

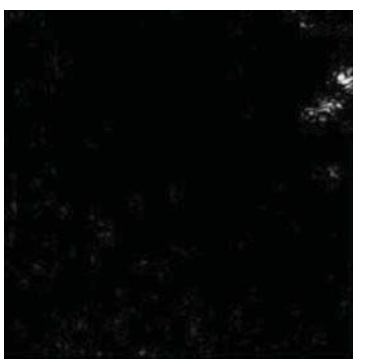

(g)

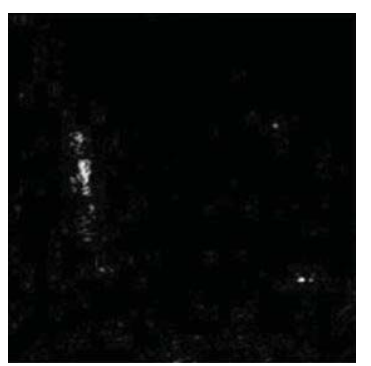

$(\mathrm{k})$

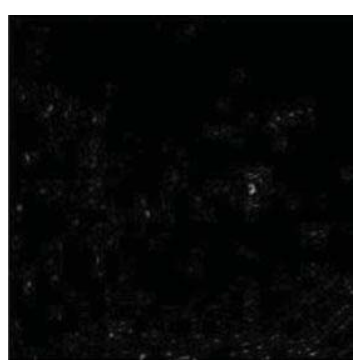

(o)

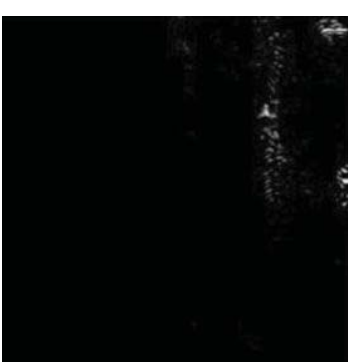

(d)

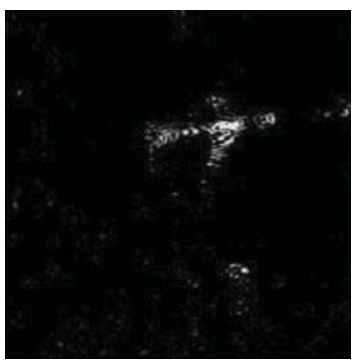

(h)

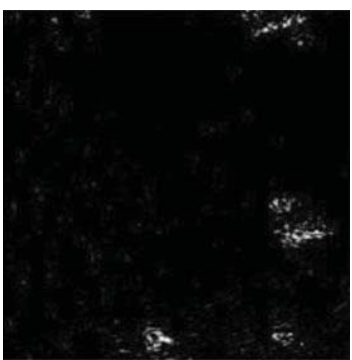

(1)

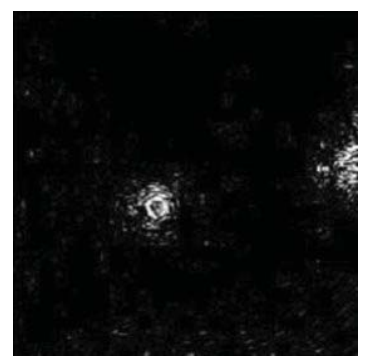

(p)

FIGURE 15: (a) microscopic image of an onion surface and (b)-(p) en face OCT images sequentially captured with a depth interval of $20 \mu \mathrm{m}$ $\left(225 \times 225 \mu \mathrm{m}^{2}\right)$.

focal plane of the objectives. A series of en face OCT images were reconstructed from top-to-bottom surfaces of the bird imprint with $1 \mu \mathrm{m}$ step. During a particular measurement instant, the instantaneous value of coefficients $a, b, c$, and $d$, which were calculated in real time are $0.83,0.92,0.90$, and 1.0 , respectively. The magnitude of the coefficients depends upon the polarization state of signal lights as well as the optical alignment condition. Using this objective, the field of view was measured at $425 \times 425 \mu \mathrm{m}^{2}(200 \times 200$ pixels $)$. The measured height of the bird imprint was around $80 \mu \mathrm{m}$. Figure 13(a) shows the area of measurement, and Figures 13 (c) to 13(d) shows the reconstructed en face OCT images of the top, middle and bottom surfaces of the coin respectively. The 3D volume rendered images with a dimension of $(x)$ $425 \times(y) 425 \times(z) 160 \mu \mathrm{m}^{3}$ is shown in Figure 14(a) with different axis of orientation.

\subsubsection{D Imaging of Biological Sample}

Onion Slice. First, we measured OCT images of an onion slice to demonstrate the feasibility for imaging biological specimen. Figure 15(a) shows the microscopic image of an onion surface using $10 \times$ and NA- 0.25 objectives to show the 


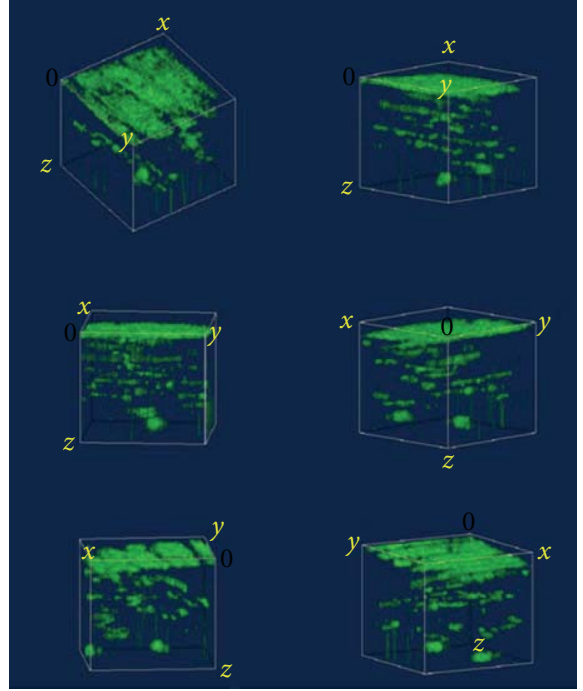

Figure 16: 3D volumetric image of the onion with a volume of 225 $\times 225 \times 280 \mu \mathrm{m}^{3}$.

cell structure. For the sample preparations, first, an index matching oil was coated on the surface of the onion slice, and then a cover glass with a thickness of $110 \mu \mathrm{m}$ was covered to avoid CCD saturations due to the surface reflection from the onion surface. The sample was set in front of the objective with a small tilt to avoid reflections from the surface of cover glass. The exposure time and the irradiation power were $7 \mathrm{~ms}$ and $224 \mu \mathrm{W}$, respectively. The OCT images were measured by scanning the reference mirror with a step resolution of $1 \mu \mathrm{m}$ up to a depth of $365 \mu \mathrm{m}$. The en face OCT images were captured reconstructed without employing any pixel binning and image averaging. The scanned depth corresponds to $280 \mu \mathrm{m}$ by considering the mean refractive index of onions as 1.3 . Figures $15(\mathrm{~b})$ to $15(\mathrm{p})$ show the sequently captured en face OCT images at different depth with a depth interval of $20 \mu \mathrm{m}$. Figure 16 shows reconstructed 3D volumetric of image $225 \times 225 \times 280 \mu \mathrm{m}^{3}(200 \times 200 \times 365$ voxel $)$ at different axis orientation. It is shown that the deeper OCT images were more blurred due to the short DF and light scatterings. However, we could resolve different cell organizations.

Diaptomus. To illustrate the system performance for imaging in vivo biological specimen, a diaptomus $[68,69]$, which is genus of copepods chosen for this study. The specimen was first kept in a slide glass with water, and then the water was extracted by ejecting with a syringe. Sequentially, a slide glass was mounted under the objective to measure the dorsal area. By decreasing the amount of water, the movement of the sample could be restricted, and then the sample position was aligned to view the dorsal area. The measurement area $\left(280 \times 320 \mu \mathrm{m}^{2}\right)$ is indicated by the black rectangular mark in Figure 17(a), which shows the dorsal view of tail part (metasome and urosome) area of the diaptomus. Figures 17(b) to 17(o) shows the reconstructed sequentially captured en face OCT images with a depth interval of $20 \mu \mathrm{m}$. Figure 18 shows the reconstructed $3 \mathrm{D}$ volumetric image of the measurement area $(280 \times 320 \times$ $350 \mu \mathrm{m}^{3}$ ). Similarly, the prosomal region of the diaptomus was also measured. Figure 19(a) shows the microscopic image of the specimen using $10 \times$ objective. Figures 19(b) to 19(1) show the reconstructed sequentially captured en face OCT images with a depth interval of $40 \mu \mathrm{m}$. Figure 20 shows the reconstructed $3 \mathrm{D}$ volumetric image of the measurement area $\left(280 \times 320 \times 360 \mu \mathrm{m}^{3}\right)(220 \times 250 \times 468$ voxel $)$ assuming that the mean refractive index of the specimen is 1.3. During measurement, the position of the sample was fixed, and then the reference mirror was scanned with a range of $468 \mu \mathrm{m}$ at a step resolution of $1 \mu \mathrm{m}$. The exposure time and the irradiation power were $1.4 \mathrm{~ms}$ and $630 \mu \mathrm{W}$, respectively. All of these en face OCT images were measured without any pixel binning and image averaging. From the reconstructed image of the prosomal region, different layered structures and the positions of some internal organs could be seen. For comparing the anatomical structures, a reconstructed crosssectional image as shown in Figure 21(b) revels the different structures, which is almost corresponds to the standard anatomy as shown in Figure 21(a). The resliced image was selected around the median region in Figure 20.

In Vivo Imaging of Biological Specimen. In this measurement, in order to demonstrate depth resolved dynamic imaging, a beating internal structure of a diaptomus in vivo has been realized. The sample was trapped in water within a glass chamber, which was fabricated with cover-glasses in a small laboratory dish. For immobilizing the specimen, the sample was partially sedated by adding very mild concentration of ethyl alcohol in the glass chamber. In this experiment, two identical water immersion objectives (Olympus, 10×, NA0.3 , LUMPlanFL 10XW) with a working distance of $3.3 \mathrm{~mm}$ were used at the both arms of the interferometer.

During measurement, looking at the en face OCT images the proper position of the sample was decided using the 2D mechanical stages. Figure 22(a) shows the in vivo microscopic view of the measurement region though the sample arm microscope. Next, the reference mirror was slowly scanned for the depth scanning, and simultaneously en face OCT images were acquired at $5 \mathrm{fps}$ with the external trigger mode. When the en face OCT image with the appropriate movement was recognized, the depth scanning was stopped at a depth of $170 \mu \mathrm{m}$ from the dorsum. The slow movements of internal organs could be seen.

Figure 18 (b)-(l) shows the in vivo en face OCT images captured sequentially at a time interval of 1 second (frame rate set was $5 \mathrm{fps}$ ). The images are captured without using any pixel binning or image averaging. This frame rate was due to the limited memory capacity of PC for archiving the images. The field of view was $280 \times 320 \mu \mathrm{m}^{2}(220 \times$ 250 pixel). The measurement time of OCT images, the exposure time and the irradiation power were $24 \mathrm{sec}, 7 \mathrm{~ms}$ and $630 \mu \mathrm{W}$, respectively. It is shown that movements of internal organs correspond to those en face OCT images. At present, the details about these movements are unclear.For practical application, the sensitivity must be enhanced to 


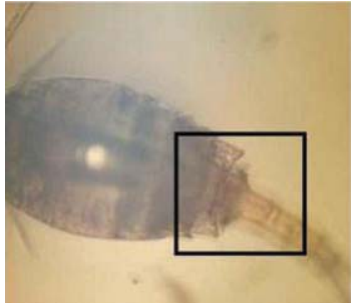

(a)

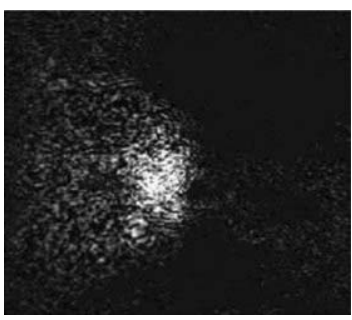

(e)

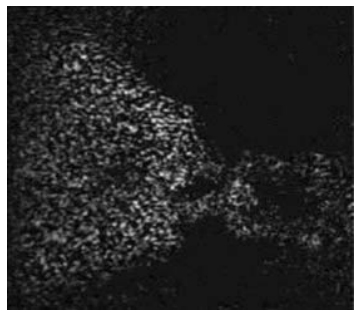

(i)

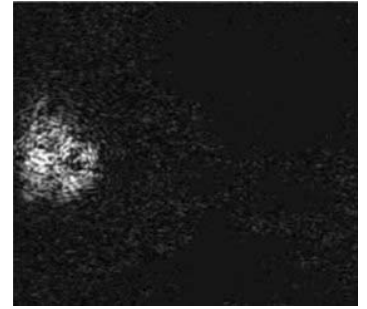

(b)

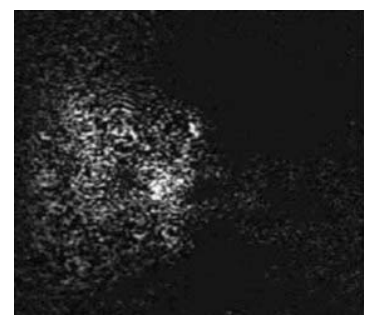

(f)

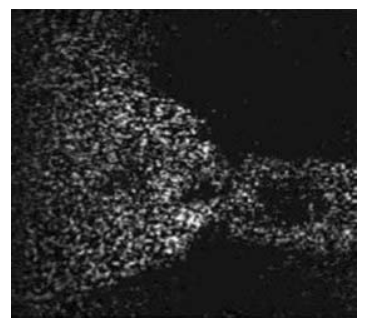

(j)

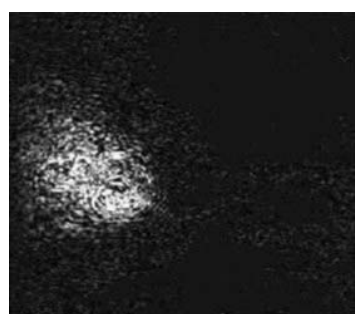

(c)

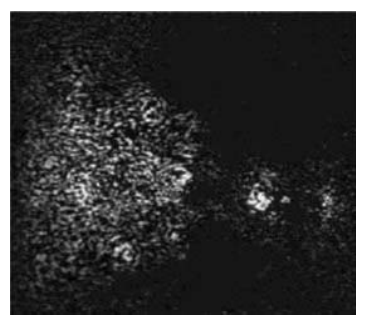

$(\mathrm{g})$

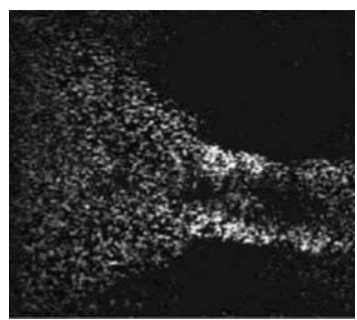

(k)

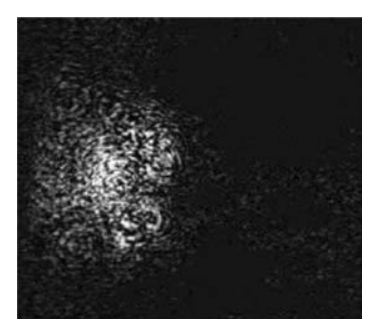

(d)

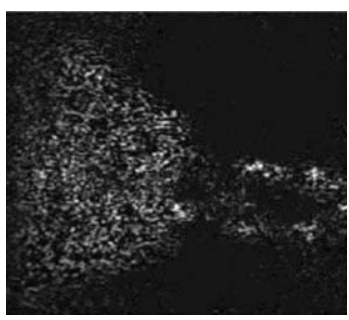

(h)

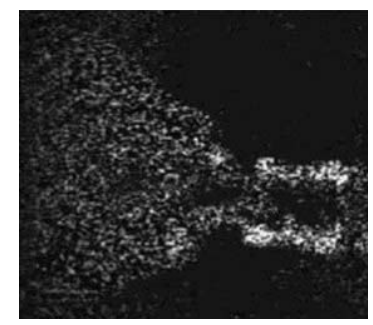

(l)

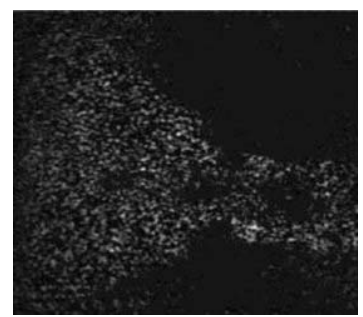

(m)

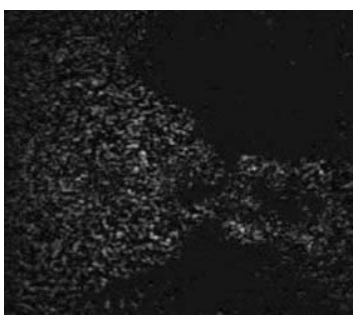

(n)

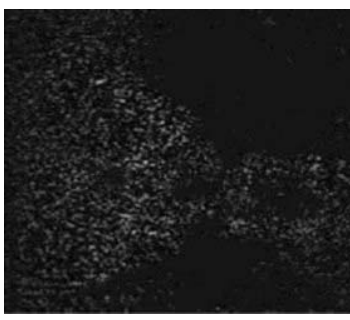

(o)

FIGURE 17: (a) microscopic image of tail part of the specimen and (b)-(o) en face OCT images sequently captured with a depth interval of $20 \mu \mathrm{m}\left(280 \times 320 \mu \mathrm{m}^{2}\right)$.

decrease the total loss for the signal light. Depending upon the application, by considering the frame rate and number of pixels in CCD, it could be possible to improve the image quality by using image averaging and pixel binning. For the enhancement of time resolution, it is essential to use camera with faster frame rate and high-power optical source with wide spectral width and to control the exposure time more precisely. Then for the axial resolution, the wavelength dispersions of optical elements in $2 \mathrm{D}$ interferometer must be compensated. Since this technique is based on polarized light, it could be also possible to measure the birefringent nature of the sample. Ultimately, further improvement of the performance of this system is more concern with the use of better-engineered optical design of the four-channel phasestepper optics. This technique would have the potential to obtain depth resolved en face images of fast phenomena in tissues, biology, and materials with short measurement time.

\section{Single-Shot FF-OCT Based on Dual-Channel Phase-Stepper Optics and 2D Analytic Signal Processing}

A novel image restoration technique for single-shot FF-OCT based on a dual-channel phase-stepper optics is introduced. A 2D analytic signal processing approach called "quaternionic analytic signal" is adopted for the reconstruction of the en face OCT image. As compared to the previously reported single-shot approaches, the main advantage of this technique is that it requires only two interferograms for the reconstruction of an en face OCT image, and thus the optical 


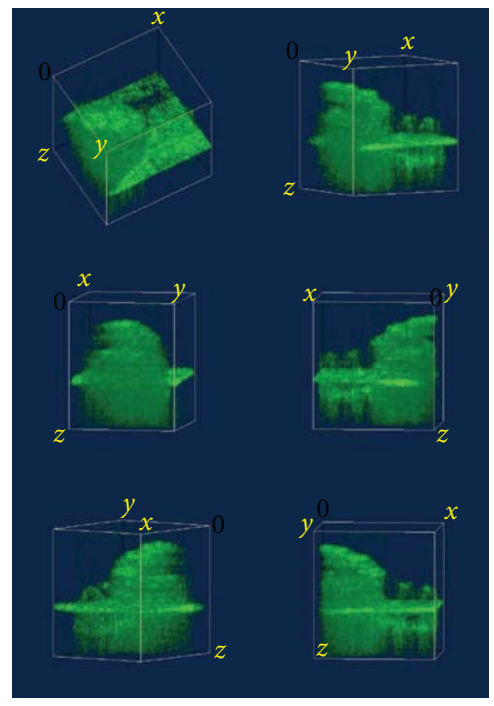

FIGURE 18: 3D volumetric image of the diaptomus with a volume of $280 \times 320 \times 350 \mu \mathrm{m}^{3}$.

complexity and alignment constrains of the system can be reduced. The technique has the following advantages over the previously reported four-channel single-shot FF-OCT systems: (i) only two interferograms are required for the reconstruction of en face OCT image; (ii) effective sampling area on the CCD can be increased; (iii) optical complexity and alignment constrains can be reduced; (iv) the overall sensitivity of the system can be improved by relative less transmission losses and other intensity aberration effects; (v) the reconstructed en face OCT image contrast is robust to the errors in relative phase shift and free from residual fringes.

6.1. Optical Scheme. A schematic of the dual-channel singleshot full-field OCT is shown in Figure 23. The system is based on a low-coherence polarization interferometer, which is followed by dual-channel phase-stepper optics. The interferometer configuration is very similar to the previously demonstrated one. After back-reflection from both arms of the interferometer, the polarization state of both the reference and sample arms is exchanged, and at the output of the interferometer, the sample beam is polarized horizontally, and the reference beam is polarized vertically. A half-wave plate (HWP-2) placed at the output of the interferometer converts the horizontal and vertical polarization into $+45^{\circ}$ and $-45^{\circ}$, respectively. A relay optics consists of two imaging lens L2 and L3 bringing the real image formed at the plane $S$ to the CCD camera through the Wollaston prism. The Wollaston prism splits the orthogonal components of both the reference and sample arm to form two $180^{\circ}$-phase-stepped images.

6.2. 2D Analytic Signal and Image Reconstruction. In signal processing, the basic idea of analytic signal is that the negative frequency components of the Fourier transform (or spectrum) of a real valued function are superfluous, due to the Hermitian symmetry of such a spectrum. In general, a signal which has no negative frequency components is called an analytic signal. The concept of analytic signal was introduced to communication theory by Gabor [70] for 1D signal. An analytic signal consists of two parts: the real part is the base signal; the imaginary (quadrature) part is the Hilbert transform (HT) of the real part. by

The Hilbert transform $f_{\mathrm{Hi}}$ of a real 1D signal $f$ is given

$$
f_{\mathrm{Hi}}(x)=f(x) * \frac{1}{\pi x},
$$

where $*$ denotes convolution. In the frequency domain, Hilbert transform is given by

$$
\begin{array}{r}
F_{\mathrm{Hi}}(u)=-i \operatorname{sign}(u) F(u) \\
\text { with } \operatorname{sign}(u)= \begin{cases}1 & \text { if } u>0 \\
0 & \text { if } u=0 \\
-1 & \text { if } u<0\end{cases}
\end{array}
$$

where $F$ and $F_{\mathrm{Hi}}$ are the Fourier transforms of $f$ and $f_{\mathrm{Hi}}$, respectively. As mentioned above, the Hilbert transform is used as a tool for phase shifting the signal by $-\pi / 2$. There is the following vivid explanation for this effect of the Hilbert transform: every signal $f$ can be represented by as a linear combination of pure frequency components $\cos (2 \pi u x+$ $\varphi$ ). The phase shifted version of this $\operatorname{sign}(u) \sin (2 \pi u x+\varphi)$ and can be derived from the cosine function by applying the operator $-(1 / 2 \pi|u|)(\partial / \partial x) \Leftrightarrow-(u /|u|)=-\operatorname{sign}(u)$. The later is identical to the transfer function of an ideal Hilbert transformer.

The analytic signal $f_{A}$ of $f$ is the sum of the original signal and the phase-shifted signal, where the phase-shifted signal is added as an imaginary part:

$$
f_{A}(x)=f(x)+i f_{\mathrm{Hi}}(x)=f(x) *\left(\delta(x)+\frac{1}{\pi x}\right) .
$$

In the frequency domain, this can be written as

$$
f_{A}(x) \Longleftrightarrow F_{A}(u)=F(u)+i F_{\mathrm{Hi}}(u)=F(u)(1+\operatorname{sign}(u)) .
$$

Thus, the analytic signal of $f$ is constructed by taking the Fourier transform $F$ of $f$, suppressing the negative frequencies and multiplying the positive frequencies by two. By applying this procedure, we do not lose any information about $f$ because of the Hermite symmetry of the spectrum of a real function.

However, the definition of analytic signal for real 2D signals is not possible by a straightforward extension of the 1D definition and without implying a preferred direction. Consequently, a number of ad hoc definitions of the $2 \mathrm{D}$ HT have been proposed [71-76] by varying degree of directionality. The typical definition have half-plane symmetry [74], quadrant based symmetry [77, 78], or rotated halfplane symmetry [79]. A recent development is the idea of extending the complex analysis of the Fourier transform to hypercomplex numbers. This concept has been proposed by Bulow and Sommer [80-82] and called hypercomplex 


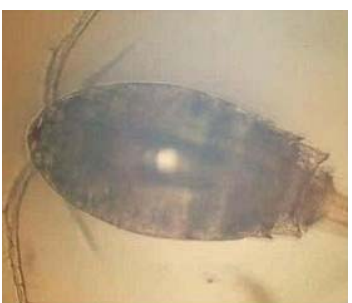

(a)

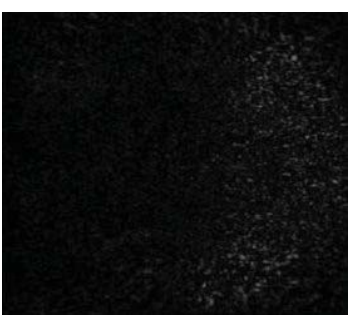

(e)

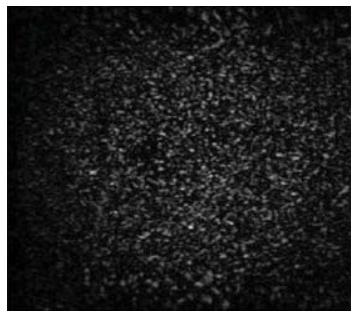

(i)

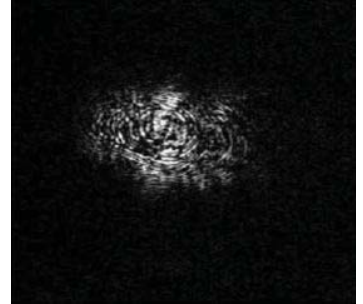

(b)

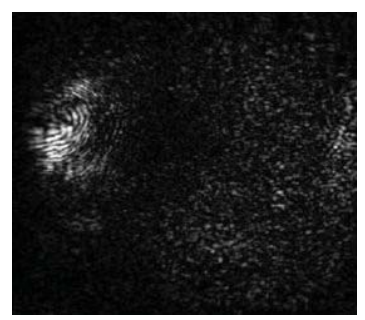

(f)

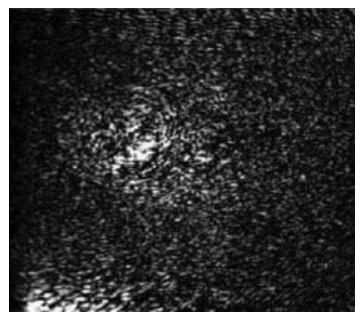

(j)

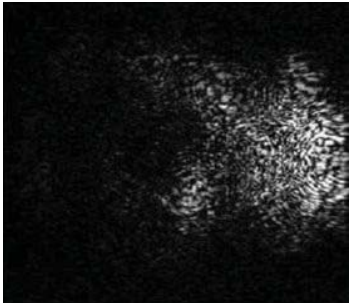

(c)

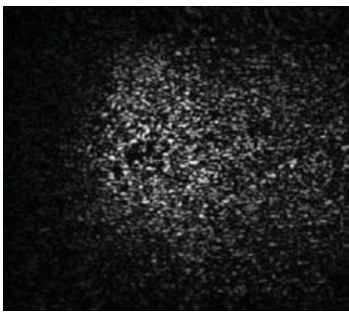

(g)

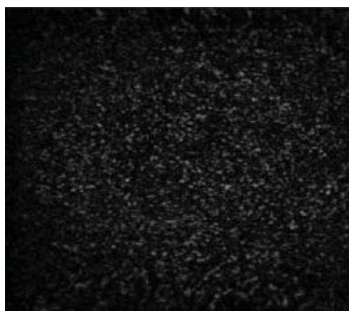

(k)

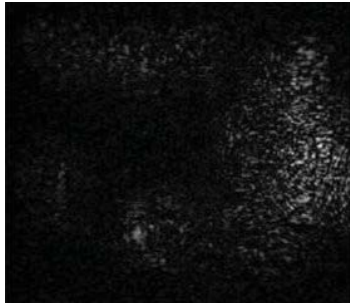

(d)

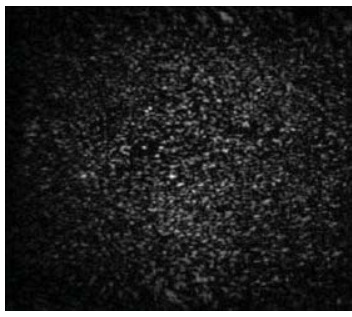

(h)

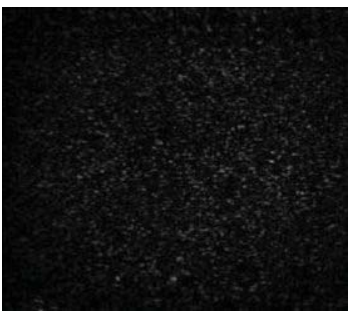

(1)

Figure 19: (a) microscopic dorsal view of the prosomal area of the diaptomus (b) to (1) en face OCT images sequently captured with a depth interval of $40 \mu \mathrm{m}\left(280 \times 320 \mu \mathrm{m}^{2}\right)$.

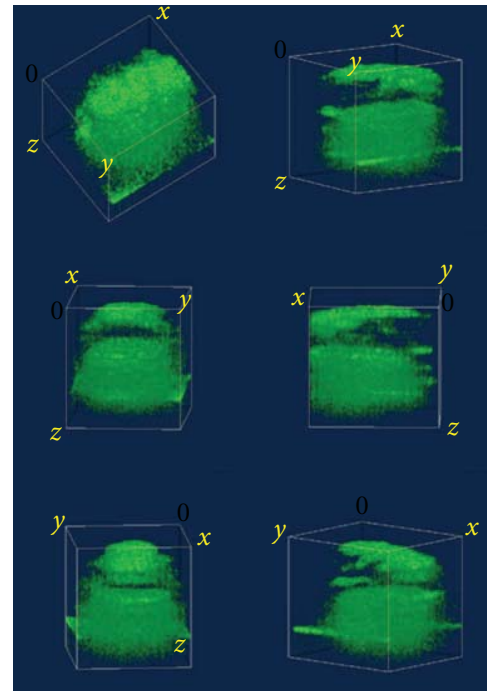

FIGURE 20: 3D volumetric image of the prosomal area of diaptomus with a volume of $280 \times 320 \times 350 \mu \mathrm{m}^{3}$.

signal representation. This approach has the potential to allow an unambiguous definition of the analytic image in 2-dimensions. This definition of analytic signal is based on quaternionic Fourier transform. The QFT was introduced in [80-85], independently. The QFT of a $2 \mathrm{D}$ signal $f(x)$ is defined as

$$
F^{q}(u)=\int_{-\infty}^{\infty} \int_{-\infty}^{\infty} e^{-i 2 \pi u x} f(x) e^{-j 2 \pi v y} d^{2} x
$$

where $u=(u, v)^{T}$ and $x=(x, y)^{T}$ are vectors and, $i$ and $j$ are the elements of the algebra of quaternions $I H=\{q=a+b i+$ $\left.c j+d k \mid a, b, c, d \in I R, i^{2}=j^{2}=-1, i j=-j i=k\right\}$. Note that the quaternionic multiplication is not commutative. The magnitude of quaternion $q=a+b i+c j+d k$ is defined as $|q|=\sqrt{q q^{*}}$, where $q^{*}=a-b i-c j-d k$ is called the conjugate of $q$.

Using the QFT the quaternionic analytic signal of a real signal in frequency domain can be written as

$$
F_{A}^{q}(u)=(1+\operatorname{sign}(u))(1+\operatorname{sign}(v)) F^{q}(u),
$$

where $F^{q}$ is the QFT of the real two-dimensional signal $f(x)$, and $F_{A}^{q}$ denotes the QFT of the quaternionic analytic signal of $f$.

In the spatial domain it can be expressed as follows:

$$
F_{A}^{q}(u)=f(x)+n \cdot f_{\mathrm{Hi}}(x),
$$




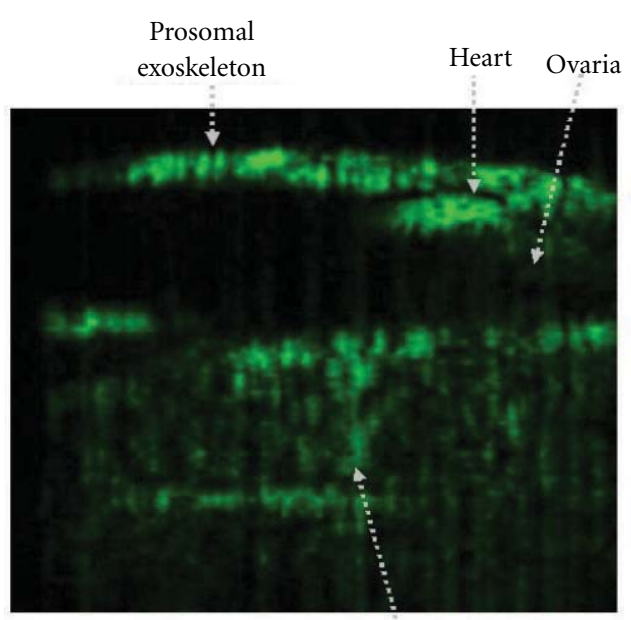

Guts with lumen

(a)

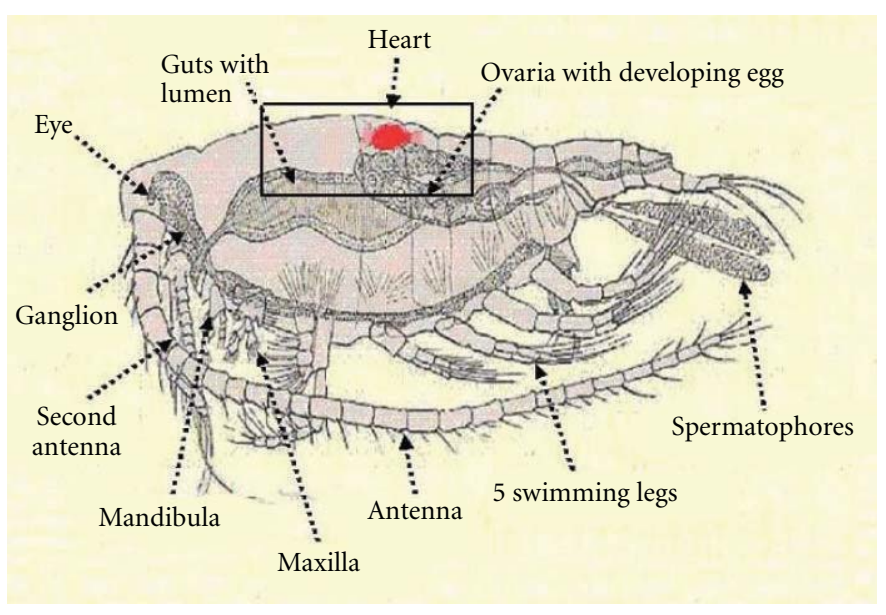

(b)

FIGURE 21: (a) resliced cross-sectional OCT image of the diaptomus $\left(320 \times 360 \mu \mathrm{m}^{2}\right)$ and (b) standard anatomy of diaptomus.

where $n=(i, j, k)^{T}$, and $f_{\mathrm{Hi}}$ is a vector which consists of the partial and the total Hilbert transform of $f$.

$$
f_{\mathrm{Hi}}(x)=\left(f_{\mathrm{Hi}_{1}}, f_{\mathrm{Hi}_{2}}, f_{\mathrm{Hi}}\right)^{T}
$$

where

$$
\begin{gathered}
f_{\mathrm{Hi}}=f * \frac{1}{\pi^{2} x y}, \quad f_{\mathrm{Hi}_{1}}=f * \frac{\delta(y)}{\pi x}, \\
f_{\mathrm{Hi}_{2}}=f * \frac{\delta(x)}{\pi y} .
\end{gathered}
$$

For image reconstruction, after extracting the interferometric component by taking the difference between the two 180-phase-shifted interferogram, two complex signals with single orthant (in the 2D case "single quadrant") spectrum is obtained as below for upper right and upper left.

For upper right:

$$
\begin{aligned}
F_{\mathrm{UR}}(u) & =\left(1+\operatorname{sign}\left(u_{1}\right)\right)\left(1+\operatorname{sign}\left(u_{2}\right)\right) \\
& =\left(F(u)-F_{\mathrm{Hi}}(u)\right)+i\left(F_{\mathrm{Hi}_{1}}(u)+F_{\mathrm{Hi}_{2}}(u)\right) .
\end{aligned}
$$

For upper left:

$$
\begin{aligned}
F_{\mathrm{UL}}(u) & =\left(1-\operatorname{sign}\left(u_{1}\right)\right)\left(1+\operatorname{sign}\left(u_{2}\right)\right) \\
& =\left(F(u)+F_{\mathrm{Hi}}(u)\right)-i\left(F_{\mathrm{Hi}_{1}}(u)-F_{\mathrm{Hi}_{2}}(u)\right)
\end{aligned}
$$

By taking the sum and difference of (37) and (39), the terms $F(u), F_{\mathrm{Hi}_{1}}(u), F_{\mathrm{Hi}_{2}}(u)$, and $F_{\mathrm{Hi}}(u)$ can be extracted individually.

Then, the en face OCT image can be reconstructed by,

$$
\mathrm{OCT}=\sqrt{f^{2}(x)+f_{\mathrm{Hi}_{1}}^{2}(x)+f_{\mathrm{Hi}_{2}}^{2}(x)+f_{\mathrm{Hi}}^{2}(x)} .
$$

\subsection{Basic Characteristics of the System}

6.3.1. Image Resolution and Sensitivity. The axial (longitudinal) resolution and transverse resolution were estimated with a standard US Air Force test pattern. The measured axial resolution was $19.8 \mu \mathrm{m}$ with a broadband superluminescent diode of spectral bandwidth $16.2 \mathrm{~nm}$ (FWHM). The lateral resolution is measured for two pairs of macroscopic objectives with numerical aperture (N. A.) of $0.1(5 \times)$ and $0.25(10 \times)$, the measured resolutions were $11 \mu \mathrm{m}$ (calculated $10.2 \mu \mathrm{m}$ ) and $4.4 \mu \mathrm{m}$ (calculated $4.1 \mu \mathrm{m}$ ), respectively. This transverse resolution not only limited by the numerical aperture of the imaging objective, but also by the CCD camera resolution.

The system sensitivity was optimized by using the minimum detectable reflectivity method, which is described in Section 5.3.2. The variation of signal intensity is measured with a US Air Force test target at the sample arm, and the optical power of the beam coming from the reference arm is attenuated with an OD filter with round trip attenuation of $40 \mathrm{~dB}$. While moving the reference arm along the optical axis in steps of $1 \mu \mathrm{m}$, successive en face images of the sample were taken. Figure 24 shows the measured sensitivity of the system, which was about $70.3 \mathrm{~dB}$ with a dynamic range of $30.3 \mathrm{~dB}$.

6.3.2. Effect of Fringe Density on the Image Reconstruction. To understand the effect of fringe density (number of fringes over the sampling pixels) on the reconstruction algorithm, a simulation is carried out by varying the fringe density over a sampling period of 200 pixels with 10 different initial phases. Since the reconstruction algorithm is based on Hilbert transform, at low fringe frequency (the signal does not vary with time or close to DC), the Hilbert transform will become zero (or at least numerically close to zero), which limits the low fringe density operation of the system. The degradation 


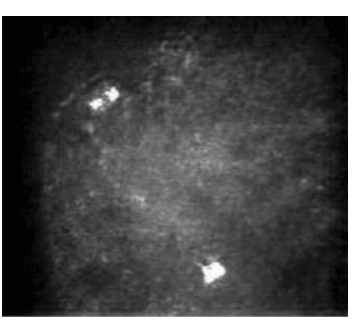

(a)

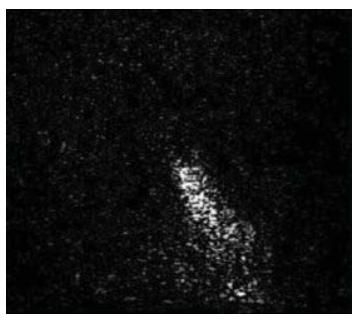

(e)

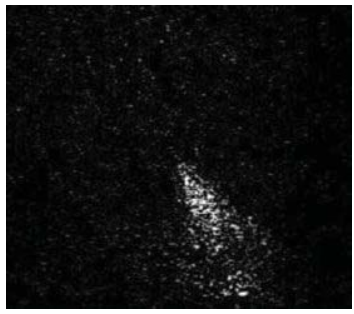

(i)

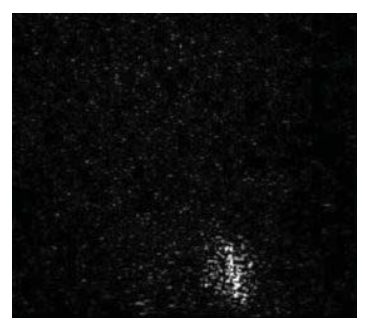

(b)

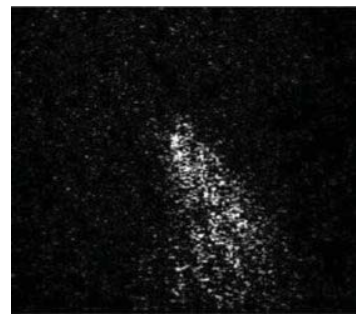

(f)

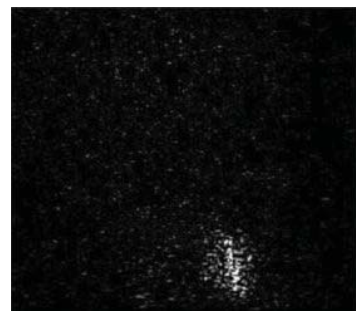

(j)

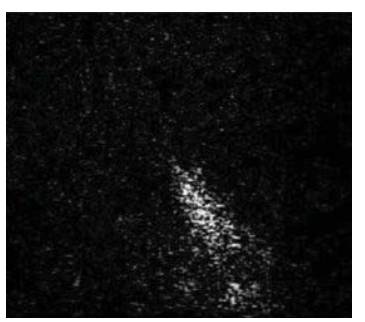

(c)

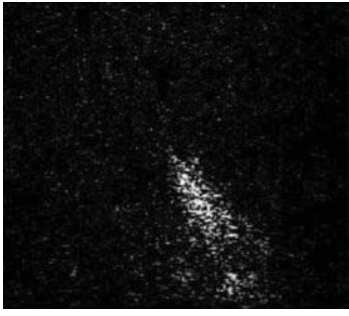

(g)

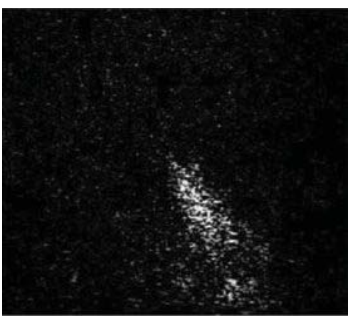

(k)

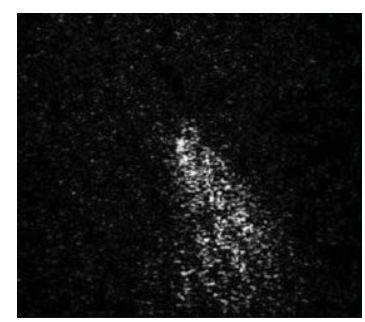

(d)

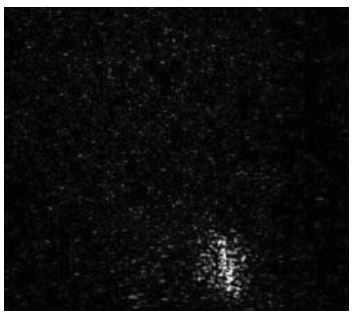

(h)

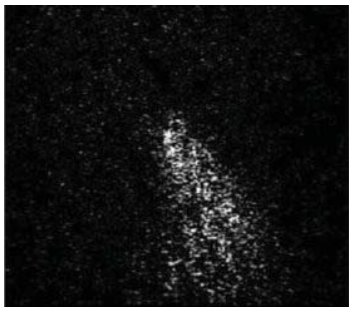

(l)

FIGURE 22: (a) in vivo image of the diaptomus through the sample arm microscopic objective and (b)-(j) in vivo en face OCT images of the moving internal structure captured at a depth of $170 \mu \mathrm{m}$ from the dorsum with a time interval of 2 seconds.

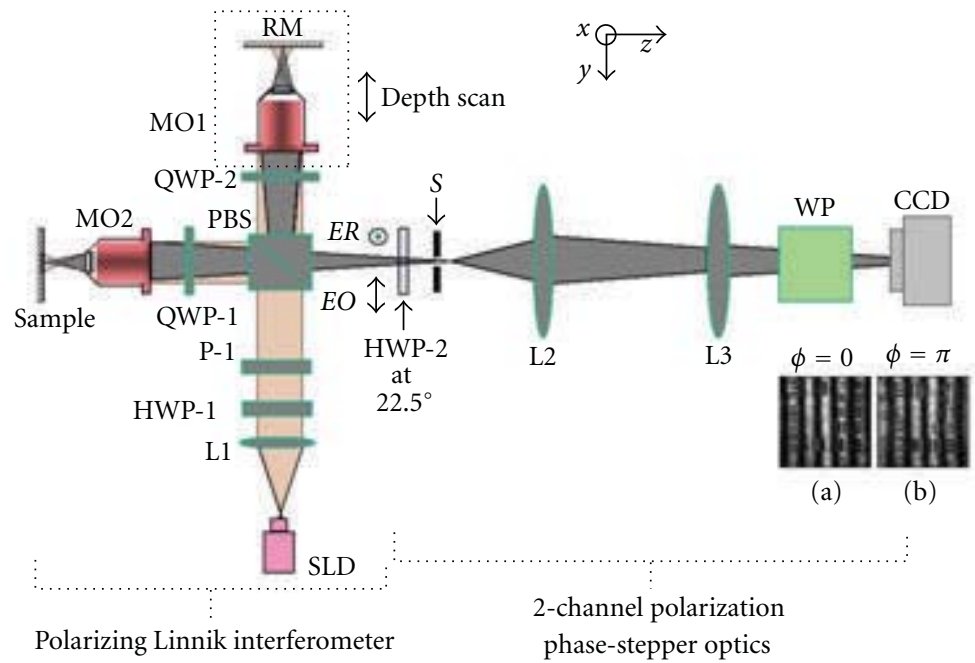

FIGURE 23: Experimental setup. L1-3: lenses, HWP: half-wave plate, P: polarizer, PBS: polarizing beam splitter, QWP: quarter wave plate, MO1-2: microscopic objectives $(0.3 \mathrm{NA} / 10 \times$ or $0.25 \mathrm{NA} / 10 \times$ or $0.1 \mathrm{NA} / 5 \times)$, RM: reference mirror, S: rectangular slit, WP: wollaston Prism, CCD: charge-coupled device. 


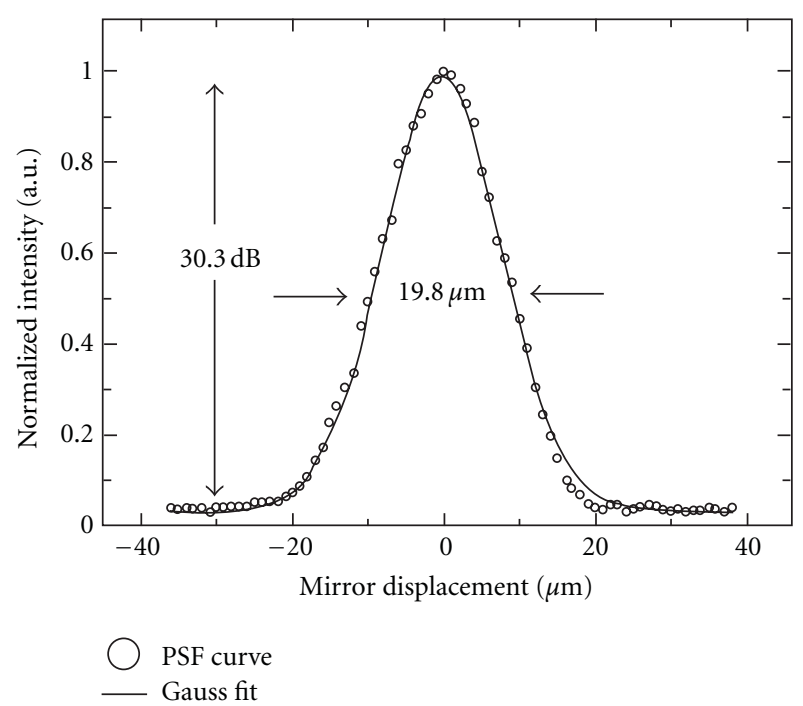

FIgURE 24: Measured axial resolution and dynamic range by displacing the reference mirror. The solid line represents the corresponding Gaussian fitting curve.

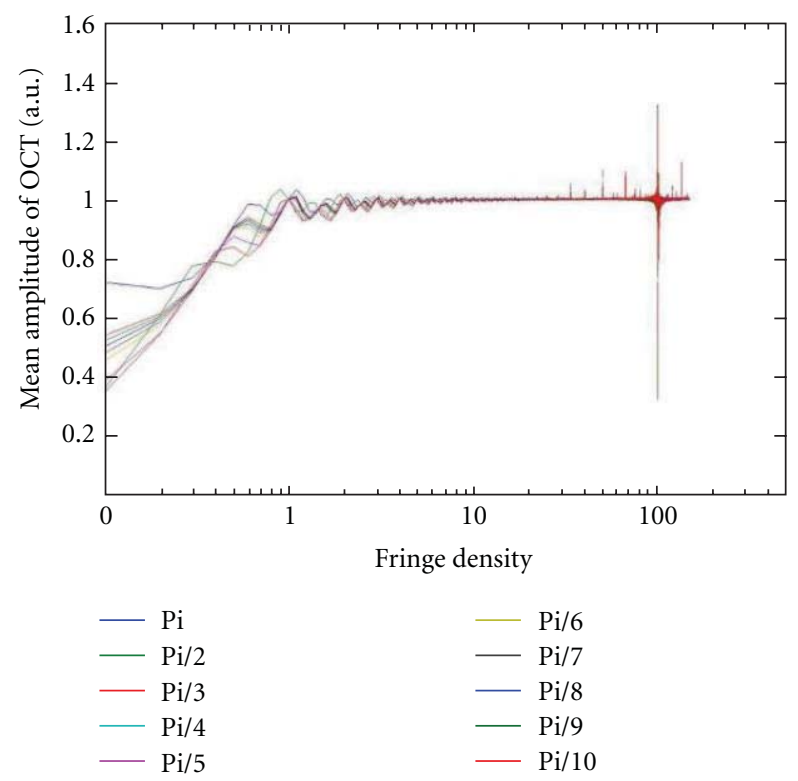

FIGURE 25: Simulation plot of fringe density versus mean amplitude of reconstructed OCT image at different initial phase of interferograms.

of OCT signal amplitude due to low fringe frequency is shown in the simulation result in Figure 25. Similarly, the number of sampling pixels limits the operation of the system at high fringe density due to the aliasing error.

\subsubsection{Effect of Relative Phase Difference on OCT Image Con-} trast. In order to verify the influence of relative phase difference between the two-phase stepped images on the contrast of the reconstructed OCT image, we simulated the OCT image contrast at various relative phase angles of Image A and Image B (Figure 23). The simulated plot is shown

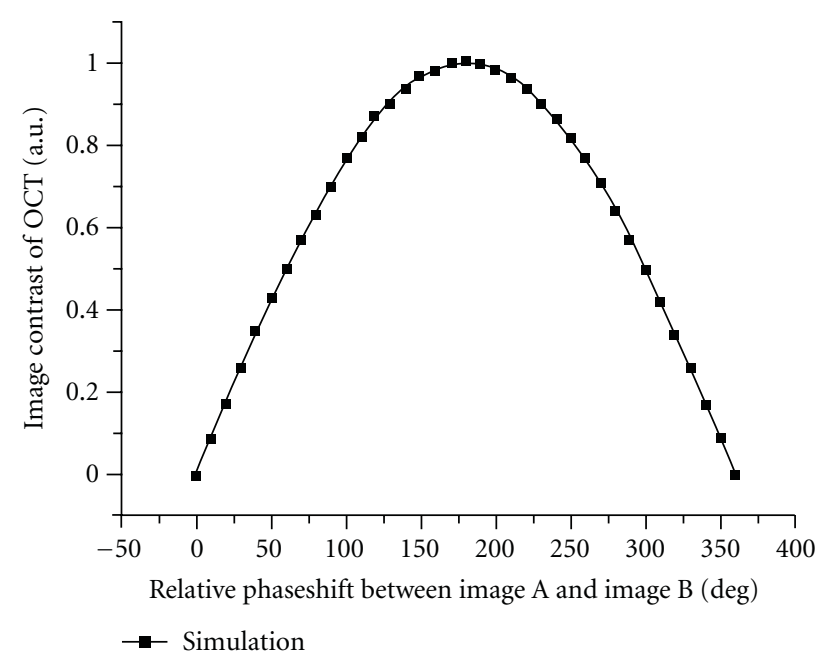

Figure 26: Simulation plot of OCT image contrast at various relative phase angles of image A and image $\mathrm{B}$. The image contrast is maximum at a phase difference of $180^{\circ}$ and degrades smoothly with the deviation from it.

in Figure 26. For experimental verification, we do not have enough mechanical freedom for changing the relative phase between Image $\mathrm{A}$ and Image $\mathrm{B}$ in the proposed experimental setup. From the simulated plot of Figure 26, it is clearly seen that the maximum signal contrast can be observed at a phase difference of $180^{\circ}$, and the signal contrast degrades smoothly in either direction when the phase shift deviates from $180^{\circ}$. Even with a phase error of $50^{\circ}$, the signal contrast is degraded by only $10 \%$ from its maximum contrast.

6.4. Imaging Result and Discussion. The feasibility of this proposed single-shot full-field OCT has been tested for imaging both rough surfaces and scattering biological specimens. Figure 27(a) shows the microscopic image of an imprint on a 10 -yen Japanese coin. The field of view was $728 \mu \mathrm{m} \times$ $850 \mu \mathrm{m}(300 \times 350$ pixels $)$. Figures $27(\mathrm{~b})$ and $27(\mathrm{c})$ shown the reconstructed en face OCT image of the top and bottom surfaces, respectively with a $5 \times$ (NA 0.1 ) objective), The exposure time and irradiation power were $1.4 \mathrm{~ms}$ and $220 \mu \mathrm{W}$, respectively. Figure $27(\mathrm{~d})$ shows the volumetric OCT image with a depth scan of $120 \mu \mathrm{m}$, the measured height from top to bottom surface was around $80 \mu \mathrm{m}$.

As a biological specimen, the tail part (metasome and urosome) of a diaptomus was imaged. Figure 28(a) shows the dorsal view of the imaging area through a microscope. Figures 28(b) and 28(c) shows the in situ en face OCT image of the urosome area of a diaptomus at a depth of 150 and $200 \mu \mathrm{m}$, respectively (including sample refractive index of 1.3). The field of view was $300 \mu \mathrm{m} \times 350 \mu \mathrm{m}(300 \times$ 350 pixels). Exposure time and irradiation power were $7 \mathrm{~ms}$ and $630 \mu \mathrm{W}$, respectively.

The $2 \mathrm{D}$ quaternionic signal processing technique is an efficient method for the extraction of en face OCT image, which needs only two $180^{\circ}$ phase-stepped interferograms. 


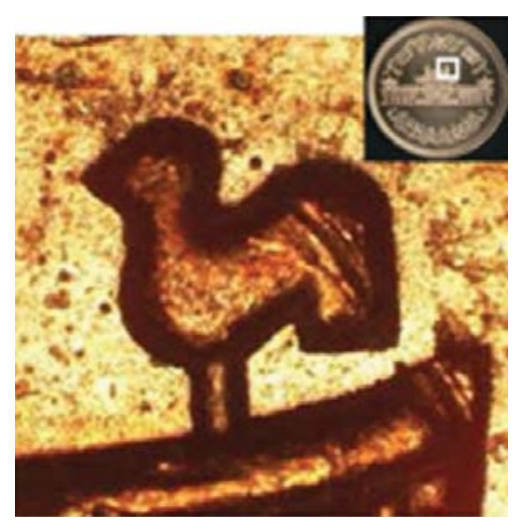

(a)

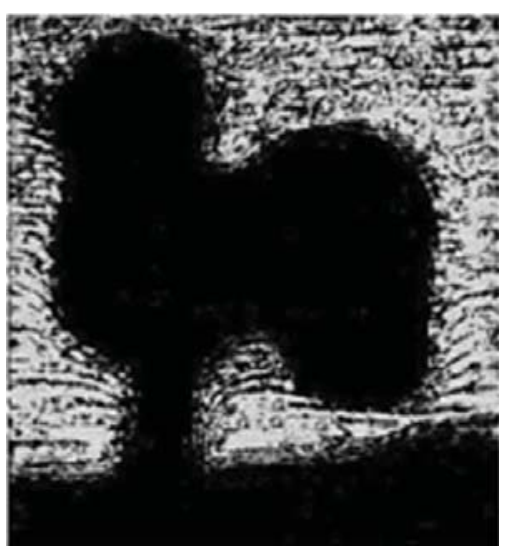

(c)

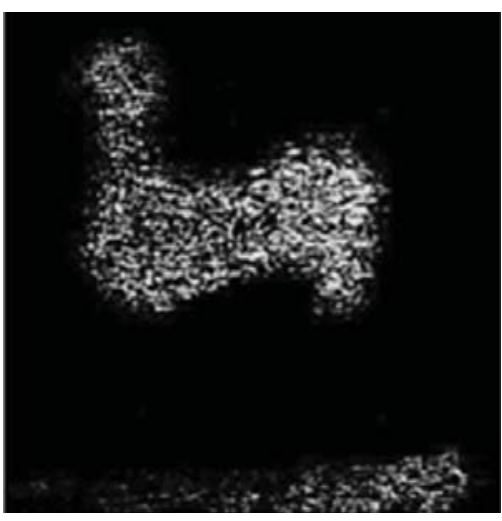

(b)

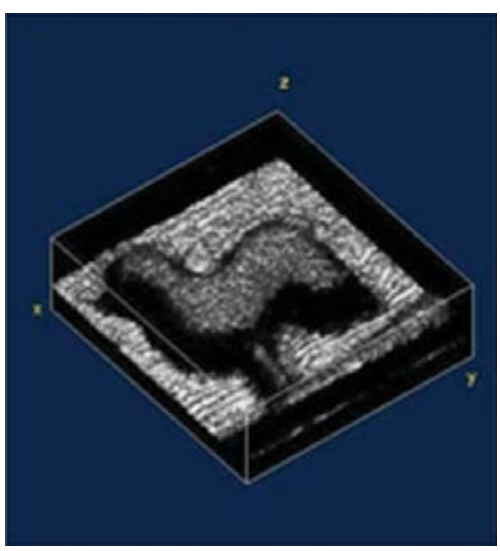

(d)

FIGURE 27: (a) microscopic image of the bird on the 10-yen Japanese coin. (b)-(c) reconstructed en face OCT images of top and bottom surfaces of the bird imprint and (d) volumetric OCT image reconstructed with 130 en face images.

Using this signal processing technique, the optical complexity of the four-channel single-shot OCT setup, which requires four interferograms could be drastically reduced with only two interferograms. Moreover, the relative less transmission losses and other intensity aberration effects could improve the overall sensitivity of the system, and the reconstructed en face OCT image contrast is robust to the errors in relative phase shift and free from residual fringes.

However, the residual unbalanced DC intensity which exists on the two-imaging channels of the phase-stepper optics due to the depolarization (on sample) effect and other sources such as pixel mismatch, and optical intensity aberration has slight influence on the contrast of the reconstructed OCT image. Further studies should be carried out to obtain a complete understanding of this system.

\section{Conclusion and Future Directions}

Full-field OCT and single-Shot FF-OCT are relatively recent alternative low-coherence imaging modalities, which can provide high-resolution, noninvasive, 3D-imaging capabilities in many areas of research, which included both fundamental and clinical applications. Its wide range of current applications ranges from medical diagnosis and surgical guidance to the characterization of polymer microstructures, which indicate that FF-OCT will play a major role in practical scientific innovation and research in years to come. Unlike conventional point scan OCT, which provide axially oriented image, FF-OCT acquires depth resolved high resolution images in the transverse direction using simple inexpensive white light source, such an arc lamp or a halogen lamp, instead of a sophisticated laser based light source. In order to improve the imaging speed and performance of FFOCT, several extensions of FF-OCT technique have been developed. Recently, LL Tech, a company based in France commercialized the first FF-OCT, which can provide cellular resolution imaging to enable noninvasive, nondestructive optical biopsies.

However, in vivo imaging is still difficult with conventional FF-OCT, which is mainly because of the sample movement and the limited imaging speed of the commercially available CCD and CMOS cameras. In order to address this problem, single-shot FF-OCT techniques have been demonstrated. The described single-shot FF-OCT technologies may enhance the abilities of current FF-OCT technique and may eventually lead to its establishment as a new scientific tool for studying fast biological phenomenon in both materials 


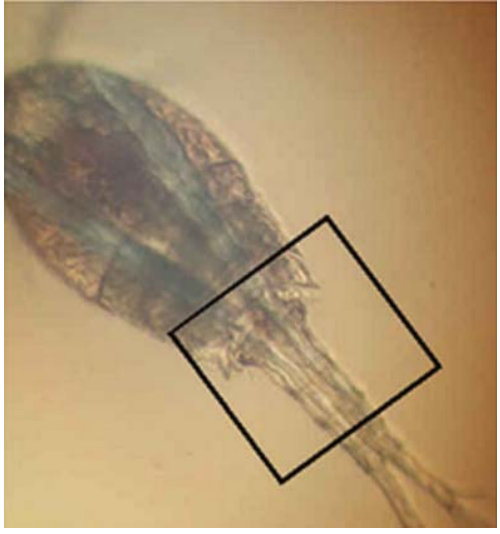

(a)

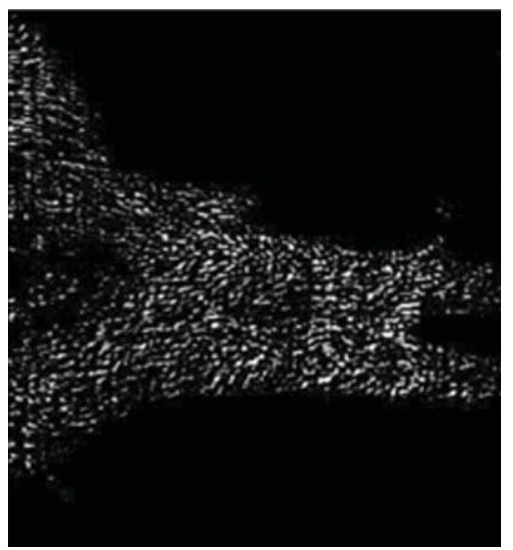

(c)

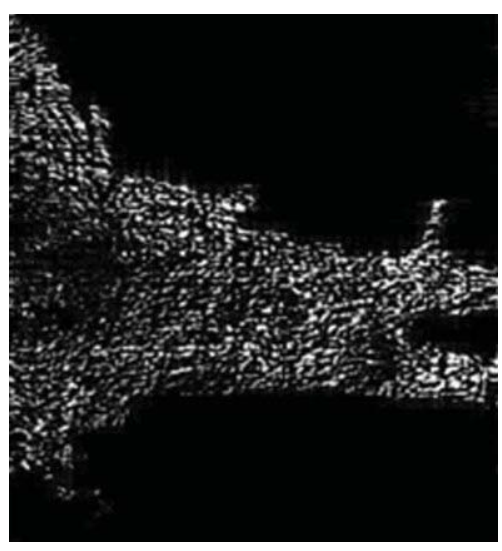

(b)

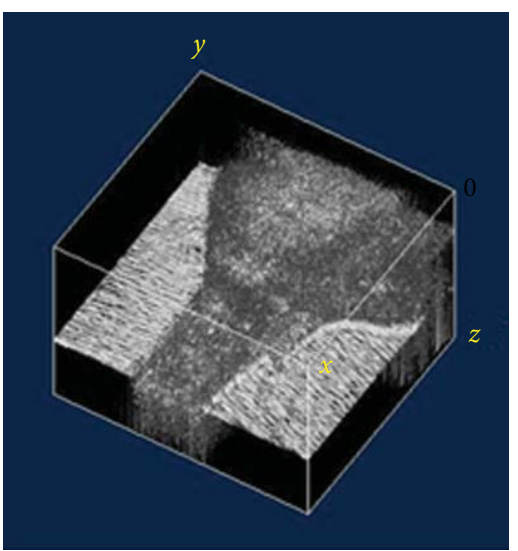

(d)

FIGURE 28: (a) microscopic image of tail part of the specimen. (b)-(c) en face OCT images at a depth of 150 and $200 \mu \mathrm{m}$, respectively (including sample refractive index of 1.3) and (c) 3D volumetric image reconstructed with 280 en face images.

and biology. The speed and performance of the system could be advanced by the use of better-engineered optical design and state-of-art-of high-power broadband optical source and high-speed detector technology. Moreover, the use of light source with longer wavelength $(1300 \mathrm{~nm})$ should improve the penetration depth in scattering medium to access more information. Since this technique is based on polarization sensitive optics, it would be possible to extend the system capabilities to provide additional information based on the polarization properties of the imaging sample. Polarizationsensitive measurements can offer enhanced contrast and specificity in structural and functional imaging. By introducing computational techniques such as 2D analytical signal approach can reduce the optical complexity of the system to some extent. Another interesting field is the development of optical contrast agents for OCT to enhance diagnostic imaging capabilities. Some of the applications that can benefit from single-shot FF-OCT include in vivo imaging of cortical neural response to cognitive and sensory stimuli for studying brain plasticity phenomenon, real-time fast imaging applications in tissue engineering, and artificial tissue technology, in developmental biology as a method to perform high-speed imaging of developing morphology and function. It can be used to study fast laser-tissue interactions such as thermal interactions, photoablation, photodisruption, and plasma-induced ablation. It can also be used for the dynamic characterization of MEMS and analyzing the complex flow dynamics in microfluidic devices. Feasibility studies should be pursued in these areas.

\section{References}

[1] N. Tanno, T. Ichikawa, and A. Saeki, "Lightwave Reflection Measurement" (Japanese), Japanese Patent \# 2010042, 1990.

[2] S. Chiba and N. Tanno, "Backscattering Optical Heterodyne Tomography, prepared for the 14th Laser Sensing Symposium" (Japanese), 1991.

[3] D. Huang, E. A. Swanson, C. P. Lin et al., "Optical coherence tomography," Science, vol. 254, no. 5035, pp. 1178-1181, 1991.

[4] A. F. Fercher, K. Mengedoht, and W. Werner, "Eye-length measurement by interferometry with partially coherent light," Optics Letters, vol. 13, no. 3, pp. 186-188, 1988.

[5] C. K. Hitzenberger, W. Drexler, and A. F. Fercher, "Measurement of corneal thickness by laser Doppler interferometry," Investigative Ophthalmology and Visual Science, vol. 33, no. 1, pp. 98-103, 1992.

[6] J. A. Izatt, M. R. Hee, E. A. Swanson et al., "Micrometer-scale 
resolution imaging of the anterior eye in vivo with optical coherence tomography," Archives of Ophthalmology, vol. 112, no. 12, pp. 1584-1589, 1994.

[7] X. Clivaz, F. Marquis-Weible, R. P. Salathe, R. P. Novak, and H. H. Gilgen, "High-resolution reflectometry in biological tissue," Optics Letters, vol. 17, pp. 4-6, 1992.

[8] J. M. Schmitt, A. Knüttel, and R. F. Bonner, "Measurement of optical properties of biological tissues by low-coherence reflectometry," Applied Optics, vol. 32, no. 30, pp. 6032-6042, 1993.

[9] G. Hausler and M. W. Lindner, "Coherence radar and spectral radar-new tools for dermatological diagnosis," Journal of Biomedical Optics, vol. 3, no. 1, pp. 21-31, 1998.

[10] A. F. Fercher, C. K. Hitzenberger, G. Kamp, and S. Y. El-Zaiat, "Measurement of intraocular distances by backscattering spectral interferometry," Optics Communications, vol. 117, no. $1-2$, pp. 43-48, 1995.

[11] R. Leitgeb, C. K. Hitzenberger, and A. F. Fercher, "Performance of fourier domain vs. time domain optical coherence tomography," Optics Express, vol. 11, no. 8, pp. 889-894, 2003.

[12] M. A. Choma, M. V. Sarunic, C. H. Yang, and J. A. Izatt, "Sensitivity advantage of swept source and Fourier domain optical coherence tomography," Optics Express, vol. 11, no. 18, pp. 2183-2189, 2003.

[13] J. F. de Boer, B. Cense, B. H. Park, M. C. Pierce, G. J. Tearney, and B. E. Bouma, "Improved signal-to-noise ratio in spectral-domain compared with time-domain optical coherence tomography," Optics Letters, vol. 28, no. 21, pp. 2067-2069, 2003.

[14] B. Považay, A. Unterhuber, B. Hermann, H. Sattmann, H. Arthaber, and W. Drexler, "Full-field time-encoded frequencydomain optical coherence tomography," Optics Express, vol. 14, no. 17, pp. 7661-7669, 2006.

[15] M. Wojtkowski, A. Kowalczyk, R. Leitgeb, and A. F. Fercher, "Full range complex spectral optical coherence tomography technique in eye imaging," Optics Letters, vol. 27, no. 16, pp. 1415-1417, 2002.

[16] R. A. Leitgeb, M. Villiger, A. H. Bachmann, L. Steinmann, and T. Lasser, "Extended focus depth for Fourier domain optical coherence microscopy," Optics Letters, vol. 31, no. 16, pp. 2450-2452, 2006.

[17] J. G. Fujimoto, "Optical coherence tomography for ultrahigh resolution in vivo imaging," Nature Biotechnology, vol. 21, no. 11, pp. 1361-1367, 2003.

[18] W. Drexler, U. Morgner, F. X. Kartner et al., "In vivo ultrahighresolution optical coherence tomography," Optics Letters, vol. 24, no. 17, pp. 1221-1223, 1999.

[19] M. E. Brezinski, G. J. Tearney, S. A. Boppart et al., "High speed catheter based OCT imaging of coronary microstructure," Circulation, vol. 94, no. 8, p. 1494, 1996.

[20] E. Beaurepaire, B. A. CLebec, L. MBlanchot, and H. SaintJalmes, "Full-field optical coherence tomography," Optics Letters, vol. 23, pp. 244-246, 1998.

[21] A. Dubois, A. C. Boccara, and M. Lebec, "Real-time reflectivity and topography imagery of depth-resolved microscopic surfaces," Optics Letters, vol. 24, no. 5, pp. 309-311, 1999.

[22] E. Bordenave, E. Abraham, G. Jortusauskas, J. Oberle, and C. Rullière, "Longitudinal imaging in biological tissues with a single-shot correlation system," Optics Express, vol. 10, no. 1, pp. 35-40, 2002.

[23] K. Grieve, A. Dubois, M. Simonutti et al., "In vivo anterior segment imaging in the rat eye with high speed white light full-field optical coherence tomography," Optics Express, vol.
13, no. 16, pp. 6286-6295, 2005.

[24] G. S. Kino and S. S. C. Chim, "Mirau correlation microscope," Applied Optics, vol. 29, no. 26, pp. 3775-3783, 1990.

[25] M. Davidson, K. Kaufman, I. Mazor, and F. Cohen, "An application of interference microscope to integrated circuit inspection and metrology," in Integrated Circuit Metrology, Inspection and Process Control, vol. 775 of Proceedings of the SPIE, pp. 233-247, 1987.

[26] A. Dubois, L. Vabre, A. C. Boccara, and E. Beaurepaire, "Highresolution full-field optical coherence tomography with a Linnik microscope," Applied Optics, vol. 41, no. 4, pp. 805812, 2002.

[27] B. E. Bouma and G. J. Tearney, Eds., Handbook of Optical Coherence Tomography, Marcel Dekker, New York, NY, USA, 2003.

[28] I. Abdulhalim, "Competence between spatial and temporal coherence in full field optical coherence tomography and interference microscopy," Journal of Optics A, vol. 8, no. 11, pp. 952-958, 2006.

[29] W. Y. Oh, B. E. Bouma, N. Iftimia, S. H. Yun, R. Yelin, and G. J. Tearney, "Ultrahigh-resolution full-field optical coherence microscopy using InGaAs camera," Optics Express, vol. 14, no. 2, pp. 726-735, 2006

[30] A. Dubois, K. Grieve, G. Moneron, R. Lecaque, L. Vabre, and C. Boccara, "Ultrahigh-resolution full-field optical coherence tomography," Applied Optics, vol. 43, no. 14, pp. 2874-2883, 2004.

[31] J. Millerd, N. Brock, J. Hayes, M. North-Morris, M. Novak, and J. Wyant, "Pixelated phase-mask dynamic interferometer," in Proceedings of the Interferometry XI, vol. 5531 of Proceedings of SPIE, pp. 304-314, Denver, Colo, USA, 2004.

[32] C. Dunsby, Y. Gu, and P. French, "Single-shot phase-stepped wide-field coherence-gated imaging," Optics Express, vol. 11, no. 2, pp. 105-115, 2003.

[33] A. F. Fercher, C. K. Hitzenberger, M. Sticker et al., "A thermal light source technique for optical coherence tomography," Optics Communications, vol. 185, no. 1-3, pp. 57-64, 2000.

[34] B. Karamata, P. Lambelet, M. Laubscher, R. P. Salathé, and T. Lasser, "Spatially incoherent illumination as a mechanism for cross-talk suppression in wide-field optical coherence tomography," Optics Letters, vol. 29, no. 7, pp. 736-738, 2004.

[35] W. Drexler, "Ultrahigh-resolution optical coherence tomography," Journal of Biomedical Optics, vol. 9, no. 1, pp. 47-74, 2004.

[36] A. F. Fercher, W. Drexler, C. K. Hitzenberger, and T. Lasser, "Optical coherence tomography-principles and applications," Reports on Progress in Physics, vol. 66, no. 2, pp. 239303, 2003.

[37] K. Creath, "Phase measurement interferometry techniques," in Progress in Optics, E. Wolf, Ed., vol. 24, pp. 349-393, Elsevier Science, Amsterdam, The Netherlands, 1988.

[38] L. Vabre, A. Dubois, and A. C. Boccara, "Thermal-light fullfield optical coherence tomography," Optics Letters, vol. 27, no. 7, pp. 530-532, 2002.

[39] E. Bordenave, E. Abraham, G. Jonusauskas et al., "Wide-field optical coherence tomography: imaging of biological tissues," Applied Optics, vol. 41, no. 10, pp. 2059-2064, 2002.

[40] M. Akiba, K. P. Chan, and N. Tanno, "Full-field optical coherence tomography by two-dimensional heterodyne detection with a pair of CCD cameras," Optics Letters, vol. 28, no. 10, pp. $816-818,2003$.

[41] M. Akiba and K. P. Chan, "In vivo video-rate cellular-level full-field optical coherence tomography," Journal of Biomedical 
Optics, vol. 12, no. 6, Article ID 064024, 2007.

[42] M. Sato, T. Nagata, T. Niizuma, L. Neagu, R. Dabu, and Y. Watanabe, "Quadrature fringes wide-field optical coherence tomography and its applications to biological tissues," Optics Communications, vol. 271, no. 2, pp. 573-580, 2007.

[43] K. Grieve, A. Dubois, M. Simonutti et al., "In vivo anterior segment imaging in the rat eye with high speed white light full-field optical coherence tomography," Optics Express, vol. 13, no. 16, pp. 6286-6295, 2005.

[44] Y. Watanabe and M. Sato, "Three-dimensional wide-field optical coherence tomography using an ultrahigh-speed CMOS camera," Optics Communications, vol. 281, no. 7, pp. 18891895, 2008.

[45] Z. P. Chen, T. E. Milner, D. Dave, and J. S. Nelson, "Optical Doppler tomographic imaging of fluid flow velocity in highly scattering media," Optics Letters, vol. 22, no. 1, pp. 64-66, 1997.

[46] D. P. Dave, T. Akkin, and T. E. Milner, "Polarizationmaintaining fiber-based optical low-coherence reflectometer for characterization and ranging of birefringence," Optics Letters, vol. 28, no. 19, pp. 1775-1777, 2003.

[47] J. F. de Boer and T. E. Milner, "Review of polarization sensitive optical coherence tomography and Stokes vector determination," Journal of Biomedical Optics, vol. 7, no. 3, pp. 359-371, 2002.

[48] D. T. Miller, J. Qu, R. S. Jonnal, and K. Thorn, "Coherence gating and adaptive optics in the eye," in Proceedings of The Coherence Domain Optical Methods and Optical Coherence Tomography in Biomedicine VII, vol. 4956 of Proceedings of SPIE, pp. 65-72, San Jose, Calif, USA, 2003.

[49] J. M. Schmitt, S. H. Xiang, and K. M. Yung, "Speckle in optical coherence tomography," Journal of Biomedical Optics, vol. 4, no. 1, pp. 95-105, 1999.

[50] W. Drexler and J. G. Fujimoto, Optical Coherence Tomography: Technology and Applications, Springer, 2008.

[51] M. S. Hrebesh, Y. Watanabe, and M. Sato, "Profilometry with compact single-shot low-coherence time-domain interferometery," Optics Communications, vol. 281, no. 18, pp. 45664571, 2008.

[52] M. S. Hrebesh, R. Dabu, Y. Watanabe, and M. Sato, "In vivo imaging of dynamic sample by real-time single-shot full- field optical coherence tomography," Optics Communications, vol. 282, no. 4, pp. 674-683, 2009.

[53] M. S. Hrebesh, Y. Watanabe, and M. Sato, "Spatial phaseshifting interferometer using paired wedge prism and combined wave plate," Japanese Journal of Applied Physics, vol. 46, no. 15, pp. L369-L371, 2007.

[54] D. Malakara, M. Servin, and Z. Malakara, Interferogram Analysis for Optical Testing, Marcel Dekker, New York, NY, USA, 1st edition, 1998.

[55] J. E. Greiven-kamp and J. H. Bruning, Optical Shop Testing, D. Malacara Ed., John Wiley \& Sons, New York, NY, USA, 2nd edition, 1992.

[56] C. Koliopoulis, "Simultaneous phase shift interferometer," in Advanced Optical Manufacturing and Testing II, vol. 1531 of Proceedings of SPIE, pp. 119-127, 1992.

[57] R. Smythe and R. Moore, "Instantaneous phase measuring interferometry," Optical Engineering, vol. 23, no. 4, pp. 361364, 1984.

[58] A. Asundi, L. Tong, and C. G. Boay, "Dynamic phase-shifting photoelasticity," Applied Optics, vol. 40, no. 22, pp. 3654-3658, 2001.

[59] N. B. E. Sawyer, S. P. Morgan, M. G. Somekh et al., "Wide field amplitude and phase confocal microscope with parallel phase stepping," Review of Scientific Instruments, vol. 72, no. 10, pp. 3793-3801, 2001.

[60] B. Barrientos, "Transient deformation measurement with ESPI using a diffractive optical element for spatial phasestepping," in Fringe, pp. 317-318, Akademie, 1997.

[61] A. Hettwer, J. Kranz, and J. Schwider, "Three channel phaseshifting interferometer using polarization-optics and a diffraction grating," Optical Engineering, vol. 39, no. 4, pp. 960-966, 2000.

[62] J. E. Millerd and N. J. Brock, "Methods and apparatus for splitting imaging and measuring wavefronts in interferometry," US Patent No. 6,304,330 and 6,522,808, 2001.

[63] B. B. Garcia, A. J. Moore, C. Perez-Lopez, L. Wang, and T. Tschudi, "Spatial phase-stepped interferometry using a holographic optical element," Optical Engineering, vol. 38, no. 12, pp. p2069-p2074, 1999.

[64] B. Kimbrough, J. Millerd, J. Wyant, J. Hayes, M. North-Morris, and M. Novak, "Low-coherence vibration insensitive Fizeau interferometer," in Proceedings of the Interferometry XIII, vol. 6292 of Proceedings of SPIE, p. 12, 2006.

[65] K. L. Baker and E. A. Stappaerts, "A single-shot pixellated phase-shifting interferometer utilizing a liquid-crystal spatial light modulator," Optics Letters, vol. 31, no. 6, pp. 733-735, 2006.

[66] B. E. A. Saleh and M. C. Teich, Fundamentals of Photonics, John Wiley \& Sons, New York, NY, USA, 1991.

[67] M. Born and E. Wolf, Principles of Optics: Electromagnetic Theory of Propagation, Interference and Diffraction of Light, chapter 13, Pergamon, Oxford, UK, 5th edition, 1975.

[68] T. W. Chad and G. Boxshall, Eds., Diaptomus Westwood, 1836, 2009.

[69] J. W. Reid, Diaptomus Restripes, 1996.

[70] D. Gabor, “Theory of communications," Journal of the Institution of Electrical Engineers, vol. 93, pp. 429-457, 1947.

[71] S. Lowenthal and Y. Belvaux, "Observation of phase objects by optically processed Hilbert transform," Applied Physics Letters, vol. 11, no. 2, pp. 49-51, 1967.

[72] H. Stark, "An extension of the Hilbert transform product theorem," Proceedings of the IEEE, vol. 59, no. 9, pp. 13591360, 1971.

[73] K. T. Eu and A. W. Lohmann, "Isotropic Hilbert spatial filtering," Optics Communications, vol. 9, no. 3, pp. 257-262, 1973.

[74] J. Ojeda-Castanada and E. Jara, "Isotropic Hilbert transform by anisotropic spatial filtering," Applied Optics, vol. 25, no. 22, pp. 4035-4038, 1986.

[75] E. Peli, "Hilbert transform pairs mechanisms," Investigative Ophthalmology \& Visual Science, vol. 30, ARVO Supplement, no. 3, p. 110, 1989.

[76] A. W. Lohmann, E. Tepichin, and J. G. Ramirez, "Optical implementation of the fractional Hilbert transform for twodimensional objects," Applied Optics, vol. 36, no. 26, pp. 66206626, 1997.

[77] Y. M. Zhu, F. Peyrin, and R. Goutte, "The use of a twodimensional Hilbert transform for Wigner analysis of 2dimensional real signals," Signal Processing, vol. 19, no. 3, pp. 205-220, 1990.

[78] S. L. Hahn, Hilbert Transforms in Signal Processing, Artech House, Norwood, Mass, USA, 1996.

[79] J. P. Havlicek, J. W. Havlicek, and A. C. Bovik, "The analytic image," in Proceedings of the IEEE International Conference on Image Processing, Santa Barbara, Calif, USA, 1997.

[80] T. Bülow and G. Sommer, "A novel approach to the 2D analytic 
signal," in Proceedings of the Computer Analysis of Images and Patterns (CAIP '99), Ljubljana, Slovenia, 1999.

[81] T. Bülow, Hypercomplex spectral signal representations for the processing and analysis of images, Ph.D. thesis, Christian Albrechts University, 1999.

[82] T. Bülow, "Hypercomplex spectral signal representations for the processing and analysis of images," Tech. Rep. 9903, Christian-Albrechts-Universität zu Kiel, Institut für Informatik und Praktische Mathematik, 1999.

[83] T. Bülow and G. Sommer, "Das Konzept einer zweidimensionalen Phase unter Verwendung einer algebraisch erweiterten Signalrepräsentation," in Mustererkennung, E. Paulus and F. M. Wahl, Eds., Tagungsband, 19. DAGM-Symposium, DAGMŽ97, pp. 351-358, Springer, 1997.

[84] T. Bülow and G. Sommer, "Multi-dimensional signal processing using an algebraically extended signal representation," in Algebraic Frames for the Perception and Action Cycle, G. Sommer and J. J. Koenderink, Eds., vol. 1315 of Lecture Notes in Computer Science, Workshop AFPACŽ 97, Kiel, pp. 148-163, Springer, Heidelberg, Germany, 1997.

[85] T. A. Ell, "Quaternion-Fourier transforms for analysis of twodimensional linear time-invariant partial differential systems," in Proceedings of the 32nd IEEE Conference on Decision and Control, pp. 1830-1841, San Antonio, Tex, USA, 1993. 

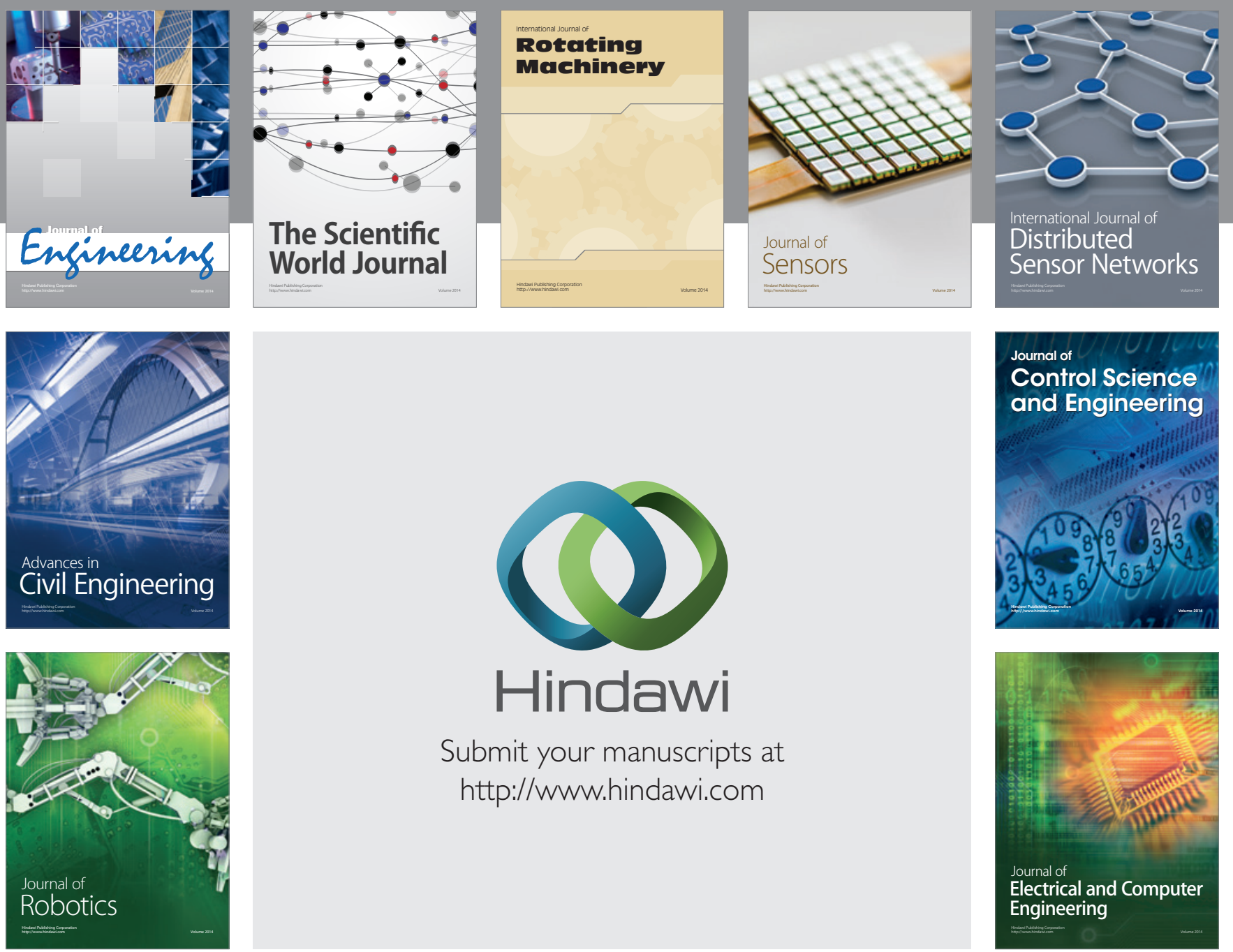

Submit your manuscripts at

http://www.hindawi.com
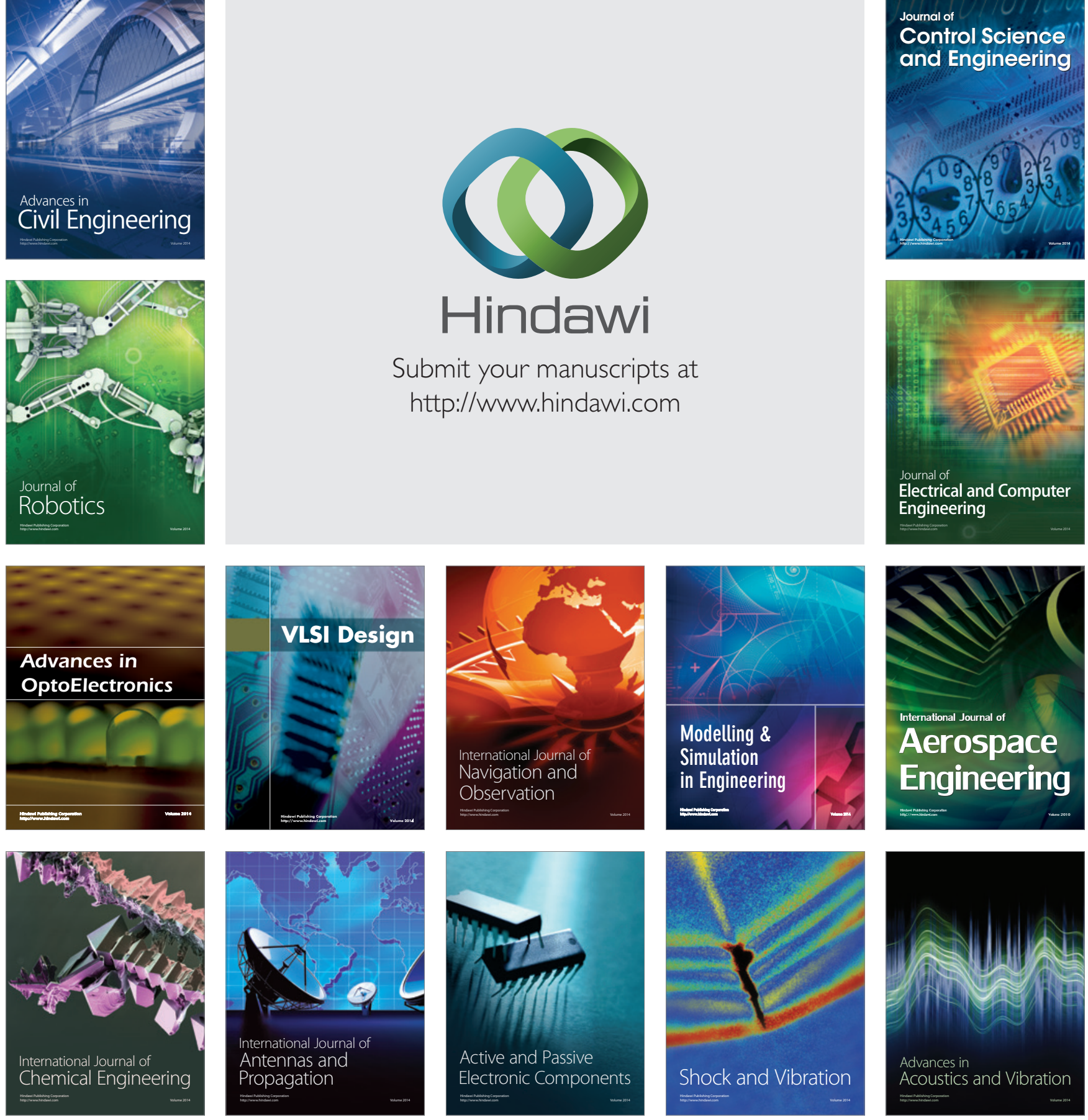